\title{
Synthesis of Isatin Derivatives Using Silver Nanoparticles as Green Catalyst: Study of Molecular Docking Interactions in SARS- CoV-2 3c-Like Protease and Determination of Cytotoxic Activities of the Compounds
}

\author{
Ponnusamy Packialakshmi, ${ }^{1}$ Perumal Gobinath,, Kaliappillai Vijayakumar, ${ }^{2}$ Daoud Ali, \\ Saud Alarifi, ${ }^{3}$ Balasubramani Ravindran, ${ }^{4}$ PitchaiSangan, ${ }^{5}$ \\ and Radhakrishnan Surendrakumar ${ }^{1}{ }^{1}$ \\ ${ }^{1} P G$ \& Research, Department of Chemistry, Nehru Memorial College (Affiliated Bharathidasan University), Puthanamapatti, \\ Tamil Nadu, India \\ ${ }^{2}$ Department of Chemistry, M. Kumarasamy College of Engineering, Karur, Tamil Nadu, India \\ ${ }^{3}$ Department of Zoology, College of Science, King Saud University, P.O. Box 2455, Riyadh, 11451 Riyadh, Saudi Arabia \\ ${ }^{4}$ Department of Environmental Energy and Engineering, Kyongi University, Suwon 16227, Republic of Korea \\ ${ }^{5}$ Hyprocell Biotech Company, 53 East Industrial Road, Branford, Connecticut 06405, USA
}

Correspondence should be addressed to Radhakrishnan Surendrakumar; surendrakumar@nmc.ac.in

Received 12 May 2021; Accepted 26 August 2021; Published 4 October 2021

Academic Editor: A. Madhan Kumar

Copyright (C) 2021 Ponnusamy Packialakshmi et al. This is an open access article distributed under the Creative Commons Attribution License, which permits unrestricted use, distribution, and reproduction in any medium, provided the original work is properly cited.

The coronavirus disease (COVID-19) is caused by the severe acute respiratory syndrome coronavirus-2 (SARS-CoV-2). As isatin-containing compounds exhibit several remarkable biological activities, isatin derivatives were prepared to combat the global pandemic caused by SARS-CoV-2 that is gripping the world. Herein, the synthesis of novel isatin derivatives has been reported. The cytotoxic activities of the compounds were determined using cancer cell (MCF-7) and normal cell (MCF-10A and MRC-5) lines. In silico molecular docking experiments were conducted using AutoDock Vina. We have successfully predicted the binding energies and the number of hydrogen bonds present. We have also identified the residues involved in hydrogen bond formation. The target compounds were synthesized using Schiff base following cyclization and Knoevenagel condensation reactions. We have focused on the recyclable synthesis of silver nanoparticles (AgNPs) using the extracts obtained from Dipteryx odorata. The extracts were used to reduce silver ions for the production of AgNPs. The synthesized nanoparticles exhibited excellent catalytic activities during the synthesis of isatin derivatives in ethanol. The formation of the target isatin derivatives has been confirmed using the Fourier transforminfrared (FT-IR), proton nuclear magnetic resonance $\left({ }^{1} \mathrm{H}\right.$ NMR) spectroscopy, ${ }^{13} \mathrm{C}$ NMR spectroscopy, mass spectrometry, and elemental analysis techniques. Compound $3 \mathrm{e}$ was found to be the most active compound when tested against the MCF-7 cancer cell line $\left(\mathrm{IC}_{50}=20.5 \mu \mathrm{M}\right)$. The activity was comparable to the activities of standard doxorubicin and other compounds. In silico molecular docking experiments were conducted to study the spike protein in SARS-CoV-2 (PDB ID: 6LU7). Compound $3 \mathrm{c}$ exhibited high binding ability $(-9.4 \mathrm{kcal} / \mathrm{mol})$. The inhibition ability was studied using hydroxychloroquine as a standard. Results from docking studies revealed that the inhibition ability of compound $3 \mathrm{c}$ was higher than the inhibition abilities exhibited by other compounds. The synthesized compound $3 \mathrm{e}$ is a potential antiviral drug that can be used for treating the COVID-19 disease. 


\section{Introduction}

The coronavirus disease (COVID-19) is caused by the severe acute respiratory syndrome coronavirus-2 (SARS-CoV-2, a novel coronavirus). SARS-CoV-2 attracted the attention of the World Health Organization (WHO) in the early part of 2003, though the presence of the virus was first detected in China in November 2002. SARS-CoV-2 is a virus that causes pneumonia. The WHO declared on the 31st of December (2019) that an infectious disease caused by SARS-CoV-2 was spreading across Wuhan (the cultural and economic hub of central China). The disease rapidly spread across 25 countries [1] and human-to-human transmission of COVID-19 was observed [2]. The disease was declared a pandemic by WHO. When a human being infected with the virus coughs, sneezes, or talks, tiny liquid droplets are expelled from the nose and/or mouth. The disease is transmitted when a person is exposed to these droplets. The Gamaleya National Center of Epidemiology and Microbiology (the Gambia, Moscow, Russia) developed the Sputnik $\mathrm{V}$ vaccine [3]. The SARS-CoV-2 virus is a single-stranded positive-sense RNA virus with a spike protein on the surface that imparts the crown-like appearance to the virus $[4,5]$. The COVID-19 epidemic is not the first human coronavirus outbreak in the twenty-first century $[6,7]$. The genome of $\mathrm{HCoV}$ consists of single-stranded positive-sense RNA [8-11]. It is important to synthesize compounds that can be used as drugs to treat COVID-19. Green chemistry involves the use of environmentally friendly or mildly hazardous reagents for the production of chemicals. Processes that reduce or eliminate the use of dangerous reagents or solvents and provide a cost-effective synthesis method are followed in the field of green chemistry for the synthesis of target compounds [12]. Microorganisms [13] and plant extracts [14-16] play an important role in the synthesis of nanoparticles (NPs). The isatin derivatives can be used to develop drugs to combat COVID-19, and the derivatives also exhibit anticancer properties. Cancer is the second leading cause of death in the world [17-20]. Uncontrolled proliferation and growth of cells cause cancer [21-23]. A mass of cancerous cells grows to form a tumor which in some cases invade other parts of the body and metastasize [24]. Most types of tumors can be surgically removed. Chemotherapyor radiation-based treatment methods can also be used to treat cancer. Chemotherapy or other methods cannot be used to treat most types of metastatic cancers. In these cases, the cancer cells are inherently resistant to chemotherapeutics or become resistant to the medication used during treatment [25]. Several chemotherapeutic anticancer drugs directly inhibit DNA synthesis, resulting in cell death. Unfortunately, in most cases, they do not specifically target tumor cells and thus exhibit high toxicity [26-30]. Analysis of the reports published by the American Cancer Society revealed that breast cancer is the second leading cause and lung cancer is the leading cause of cancer death in women. Four different phase I trials were conducted with the compound sunitinib, but the use of the compound had to be discontinued as it exhibited high activity [31-34]. The biological activities of the isatin derivatives are demonstrated in
Figure 1 [35-37]. Methisazone (isatin-thiosemicarbazone) functions as a prophylactic agent and is used to treat various viral diseases [38]. Isatin and 2-thioxo-imidazolidine-4-one derivatives exhibit excellent antiviral, anticancer, and other pharmacological activities. Herein, the synthesis and characterization of silver NPs (AgNPs) have been reported. The NPs were used as effective and highly efficient green catalysts during the preparation of isatin-based thiosemicarbazone conjugates.

The 2-thioxo-imidazolidine-4-one moiety is also present in our newly synthesized compound and the sulfur analog of hydantoin, in which one carbonyl group is substituted by a thiocarbonyl group [39]. Thioxoimidazolidine derivatives show anticancer, anti-inflammatory, and antiviral activities in Figure 2 [40-44].

Based on the evidence presented above, new medicines for the treatment of killer diseases such as antiviral and anticancer activities are urgently needed. The cytotoxic activities were determined, and the in silico molecular docking technique was used to study the effect of the compounds on the spike proteins of SARS-CoV-2.

\section{Materials and Methods}

2.1. General Methods. The chemicals, such as isatin, thiosemicarbazide, and various aldehydes, were purchased from Sigma-Aldrich with $99 \%$ purity. Melting points are recording in open capillary tubes and were uncorrected. On a Shimadzu 8201 pc, the FT-IR spectra were reported using the $\mathrm{KBr}$ pellet process $\left(4000-400 \mathrm{~cm}^{-1}\right)$. An Avance Bruker DRX-300 MHz spectrometer was used for NMR $\left({ }^{1} \mathrm{H}-\mathrm{NMR}\right.$ and ${ }^{13} \mathrm{C}-\mathrm{NMR}$ ) analysis using $\mathrm{CDCl}_{3}$ as a solvent. The mass spectrum was recorded by the PerkinElmer GCMS model Clarus SQ 8 (EI). The morphology of silver nanoparticles is confirmed by using SEM and TEM. For SEM research, a Scanning Electron Microscope (SEM) model VP-1450 (LEO, Co., Germany) was used. For transmission electron microscopy (TEM) analysis, an LEO 912 AB instrument was used. Elemental analyses $(\mathrm{C}, \mathrm{H}$, and $\mathrm{N})$ were performed using an elemental analyzer (Version EL III). The purity of the compound was determined by using thin-layer chromatography (TLC) with a silica gel plate.

2.2. Preparation of Dipteryx odorata Extract. A $7 \mathrm{~g}$ of dried fresh powder of Dipteryx odorata was mixed with $200 \mathrm{ml}$ of water and stirred for 45 minutes on a magnetic stirrer to make an aqueous extract. After boiling for 30 minutes, the mixture was strained, cooled, and filtered. The boiled extract was kept refrigerated and used in subsequent experiments.

2.2.1. Synthesis of AgNPs. The $25 \mathrm{ml}$ of $2 \mathrm{mM}$ silver nitrate solution was stored in an RB flask, and Dipteryx odorata extract was added for reduction into $\mathrm{Ag}^{+}$ions. The mixture, which included silver nitrate solution and plant extracts, was stirred and heated for 52 hours at $80^{\circ} \mathrm{C}$ with $600 \mathrm{rpm}$. The color of the solution changed from yellow to dark brown. The nanoparticle solution was purified by repeated centrifugation at 12,000 rpm for 30 minutes and then decanted and 


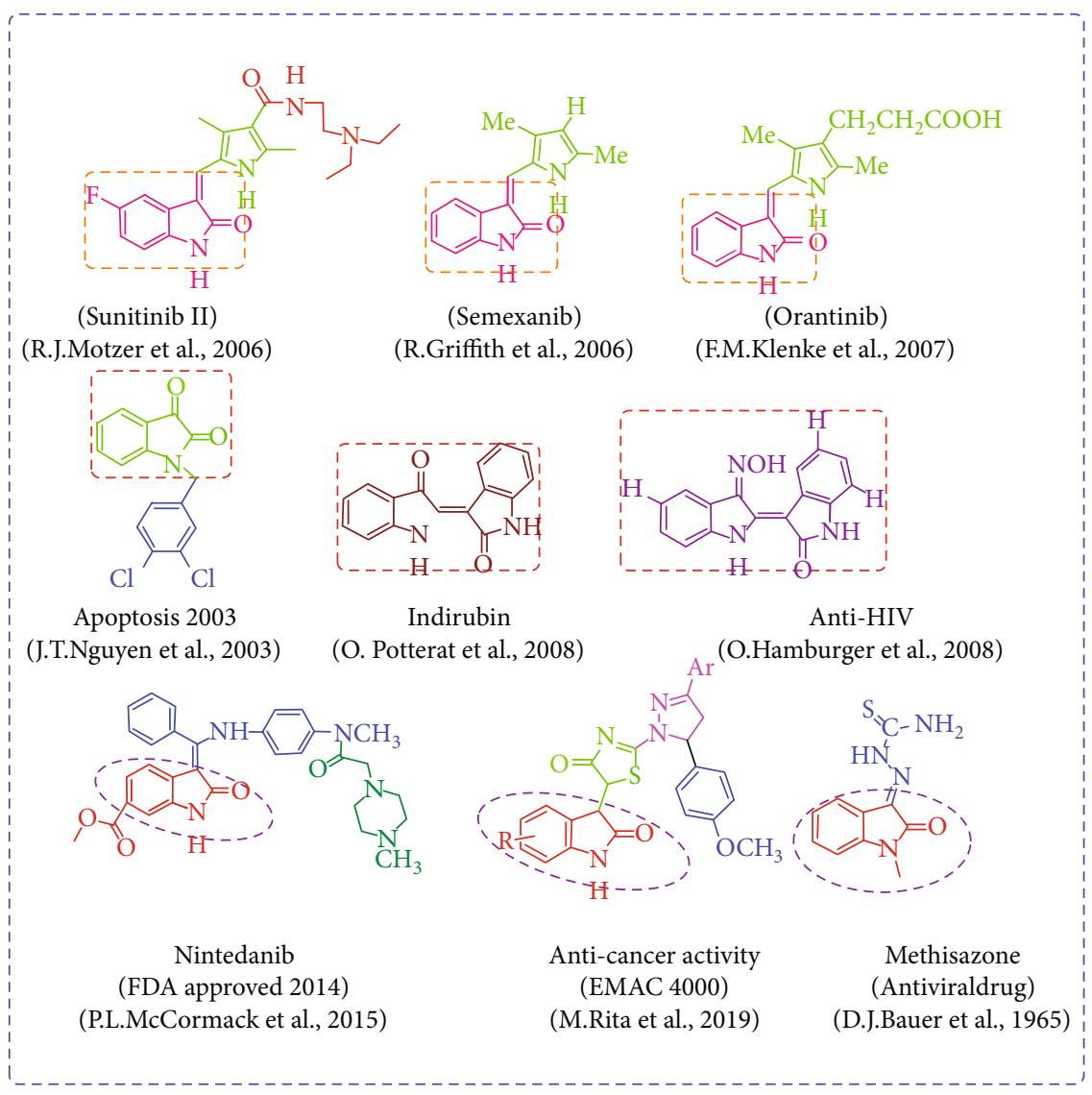

FIgURE 1: Biologically active compounds of isatin derivatives.

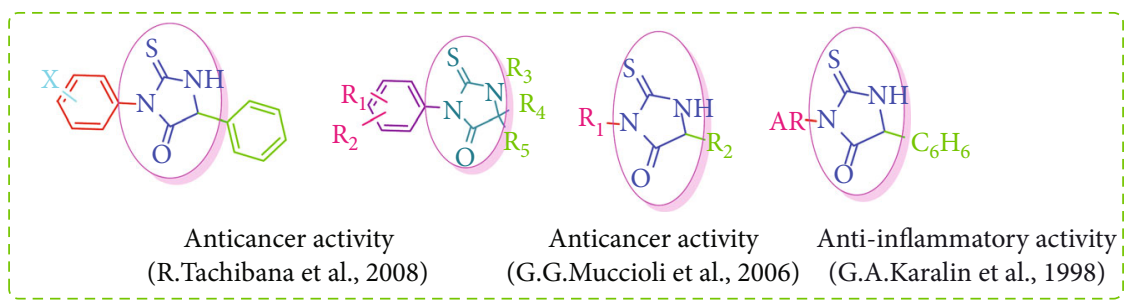

FIGURE 2: Anticancer and anti-inflammatory or biological activities of 2-thiohydantoin derivatives.

dried in an oven at $70^{\circ} \mathrm{C}$ overnight. The synthesis of AgNPs is presented in Scheme 1.

2.3. General Process for the Synthesis of Compound 1. Isatin $(2.94 \mathrm{~g}, 0.02 \mathrm{~mol})$, thiosemicarbazide $(3.64 \mathrm{~g}, 0.04 \mathrm{~mol})$, and green catalyst AgNPs were refluxed for $2 \mathrm{~h}$ to give compound 1. TLC was used to track the reaction, which used the eluting solvents hexane:ethyl acetate $(1: 2)$, until the sample was purified and washed with water before being dried to yield the crude sample. The crude sample dissolved in DMF and centrifuged to separate the catalyst and later the catalyst was washed several times with $\mathrm{EtOH}$, then dried and reutilized three times for the same reaction. Then, the product was recrystallized with ethanol. Yellow solid; yield: $98 \%$; mp: $244^{\circ} \mathrm{C}$; IR (KBr) $3475\left(\mathrm{NH}_{2}\right), 3421$ (NHstr), 3143 (ArCHstr), 1682 (C=Nstr), 1496 (Ar-C=C) $\mathrm{cm}^{-1} ;{ }^{1} \mathrm{H}$ NMR $\left(300 \mathrm{MHz}, \mathrm{CDCl}_{3}\right), \delta 12.85(\mathrm{~s}, 2 \mathrm{H}$, thiosemicarbazide $=\mathrm{N}$ $\mathrm{NH}), 11.24(\mathrm{~s}, 1 \mathrm{H}$, isatin-NH), $7.53(\mathrm{~d}, 1 \mathrm{H}$, isatin, $J=7.5$ $\mathrm{Hz}), 7.32(\mathrm{t}, 1 \mathrm{H}$, isatin, $J=7.7 \mathrm{~Hz}), 7.06(\mathrm{t}, 1 \mathrm{H}$, isatin, $J=$ $7.2 \mathrm{~Hz}), 6.92(\mathrm{~d}, 1 \mathrm{H}$, isatin, $J=7.8 \mathrm{~Hz}), 6.89(\mathrm{~s}, 4 \mathrm{H}$, thiosemicarbazide- $\left.\mathrm{NH}_{2}\right) ;{ }^{13} \mathrm{C} \mathrm{NMR}\left(300 \mathrm{MHz}, \mathrm{CDCl}_{3}\right) \delta 176.84$ (2C, C-2,3, C=S), 142.62 (2C, C-1,4, C=N), 142.50 (1C, C10, isatin), 132.72 (1C, C-8, isatin), 131.30 (1C, C-6, isatin), 122.45 (1C, C-9, isatin), 119.73 (1C, C-7, isatin), 111.22 (1C, C-5, isatin). EI-MS (relative intensity \%): $\mathrm{m} / \mathrm{z} 293.05\left(\mathrm{M}^{+}\right.$, $10.9 \%)$. Elemental analysis $\left(\mathrm{C}_{10} \mathrm{H}_{11} \mathrm{~N}_{7} \mathrm{~S}_{2}\right)$ : calculated $\mathrm{C}$, 40.94, H, 3.78, N, 33.42; found C, 40.96, H, 3.80, N, 33.45 .

2.3.1. General Process for the Synthesis of Compound 2. The compound 1 ( $2.9 \mathrm{~g}, 0.01 \mathrm{~mol})$ underwent cyclization with chloroethyl acetate $(2.14 \mathrm{ml}, 0.02 \mathrm{~mol})$, fused sodium acetate $(0.82 \mathrm{~g}, 0.01 \mathrm{~mol})$, and AgNPs used as a catalyst and refluxed 


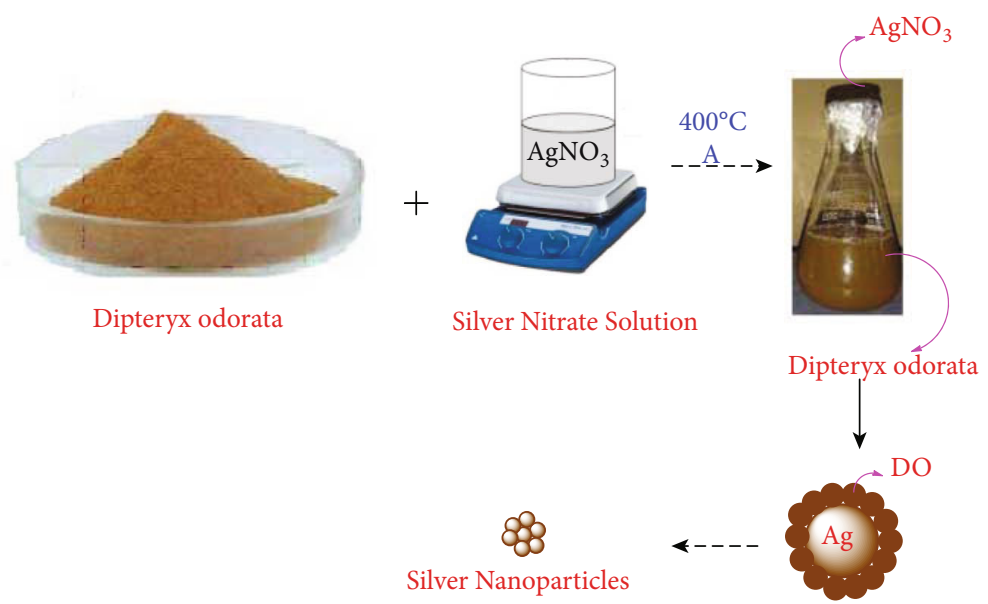

Scheme 1: Synthesis of silver nanoparticles from extract of Dipteryx odorata.

for $3 \mathrm{~h}$ to give compound 2 . The reaction was checked by TLC with eluting solvents hexane: ethyl acetate $(1: 2)$. The obtained product was recrystallized with ethanol. Brown color solid; Yield: 95\%; mp; $236^{\circ} \mathrm{C}$; IR ( $\left.\mathrm{KBr}\right) 3421$ (NHstr), 3143 (Ar-CHstr), 1699 (C=O), 1682 (C=Nstr), 1496 (Ar$\mathrm{C}=\mathrm{C}), 1135(\mathrm{C}=\mathrm{S})\left(\mathrm{cm}^{-1}\right) ;{ }^{1} \mathrm{H}$ NMR $\left(300 \mathrm{MHz}, \mathrm{CDCl}_{3}\right), \delta$ 12.89 (s, $2 \mathrm{H}$, imidazolidine- $\mathrm{NH}), 10.68(\mathrm{~s}, 1 \mathrm{H}$, isatin- $\mathrm{NH})$, $7.53(\mathrm{~d}, 1 \mathrm{H}$, isatin, $J=7.5 \mathrm{~Hz}), 7.30(\mathrm{t}, 1 \mathrm{H}$, isatin, $J=7.7$ $\mathrm{Hz}), 7.06(\mathrm{t}, 1 \mathrm{H}$, isatin, $J=7.4 \mathrm{~Hz}), 6.92(\mathrm{~d}, 1 \mathrm{H}$, isatin, $J=$ $7.8 \mathrm{~Hz}), \quad 2.75$ (s, $4 \mathrm{H}$, imidazolidine- $\left.\mathrm{CH}_{2}\right) ;{ }^{13} \mathrm{C} \quad \mathrm{NMR}$ $\left(300 \mathrm{MHz}, \mathrm{CDCl}_{3}\right) \delta 179.61(2 \mathrm{C}, \mathrm{C}-4,7, \mathrm{C}=\mathrm{S}), 162.93(2 \mathrm{C}$, $\mathrm{C}-2,5, \mathrm{C}=\mathrm{O}), 145.75$ (1C, C-14, isatin), 142.53 (2C, C-1,8, $\mathrm{C}=\mathrm{N}), 132.79$ (1C, C-12, isatin), 131.33 (1C, C-6, isatin), 122.48 (1C, C-13, isatin), 119.79 (1C, C-11, isatin), 111.22 (1C, C-9, isatin), 39.13 (2C, C-3,6); EI-MS (relative intensity $\%): \quad \mathrm{m} / \mathrm{z} \quad 373.04 \quad\left(\mathrm{M}^{+}, 19.3 \%\right)$. Elemental analysis $\left(\mathrm{C}_{14} \mathrm{H}_{11} \mathrm{~N}_{7} \mathrm{O}_{2} \mathrm{~S}_{2}\right)$ : calculated $\mathrm{C}, 45.03, \mathrm{H}, 2.97, \mathrm{~N}, 26.26$; found $\mathrm{C}, 40.05, \mathrm{H}, 2.99, \mathrm{~N}, 26.28$.

2.3.2. General Process for the Synthesis of Compound $3 a$. Compound $2(0.74 \mathrm{~g}, 0.002 \mathrm{~mol})$ was mixed with ethanol and aromatic aldehyde, and AgNPs were used as a catalyst. The mixture was refluxed for $3 \mathrm{~h}$ and recrystallized by ethanol. The reaction was confirmed by TLC with eluting solvents hexane: ethyl acetate $(1: 2)$ to give $3 \mathrm{a}$. The remaining compounds (3b-3n) were synthesized by the abovementioned procedure. Light yellowish brown color solid; yield: 90\%; mp: $230^{\circ} \mathrm{C}$; IR (KBr); 3422 (NHstr), 3170 (ArCHstr), 1673 (C=O), 1697 (C=Nstr), 1493 (Ar-C=C), 1130 $(\mathrm{C}=\mathrm{S})\left(\mathrm{cm}^{-1}\right) ;{ }^{1} \mathrm{H}$ NMR $\left(300 \mathrm{MHz}, \mathrm{CDCl}_{3}\right), \delta 12.84(\mathrm{~s}, 2 \mathrm{H}$, imidazolidine-NH), 10.65 (s, $1 \mathrm{H}$, isatin- $\mathrm{NH}), 7.73(\mathrm{~d}, 4 \mathrm{H}, J$ $=10.20, \mathrm{Ar}-\mathrm{H}), 7.56(\mathrm{~d}, 1 \mathrm{H}$, isatin, $J=7.5 \mathrm{~Hz}), 7.51(\mathrm{t}, 4 \mathrm{H}$, Ar-H), $7.39(\mathrm{t}, 2 \mathrm{H}, \mathrm{Ar}-\mathrm{H}), 7.32(\mathrm{t}, 1 \mathrm{H}$, isatin, $J=7.7 \mathrm{~Hz})$, $7.01(\mathrm{t}, 1 \mathrm{H}$, isatin, $J=7.4 \mathrm{~Hz}), 6.90(\mathrm{~d}, 1 \mathrm{H}$, isatin, $J=7.8 \mathrm{~Hz}$ ), $6.89(\mathrm{~s}, 2 \mathrm{H}$, imidazolidine $=\mathrm{CH}) ;{ }^{13} \mathrm{C}$ NMR $(300 \mathrm{MHz}$, $\left.\mathrm{CDCl}_{3}\right) \delta(\mathrm{ppm}): 179.05$ (2C, C-4,7, C=S), 162.43 (2C, C$2,5, \mathrm{C}=\mathrm{O}), 154.53(2 \mathrm{C}, \mathrm{C}-1,8, \mathrm{C}=\mathrm{N}), 145.70(1 \mathrm{C}, \mathrm{C}-14$, isatin), 142.03 (2C, C-3,6, imidazolidine-CH), 132.76 (1C, C12 , isatin), $132.25,130.81,128.26,121.98,119.31$ (14C, C$\left.3^{\prime}, 6^{\prime}, \mathrm{Ar}\right), 131.30$ (1C, C-6, isatin), 122.45 (1C, C-13, isatin),
119.89 (1C, C-11, isatin), 110.71 (1C, C-9, isatin); EI-MS (relative intensity \%): $\mathrm{m} / \mathrm{z} 549.10\left(\mathrm{M}^{+}, 30.6 \%\right)$. Elemental analysis $\left(\mathrm{C}_{28} \mathrm{H}_{19} \mathrm{~N}_{7} \mathrm{O}_{2} \mathrm{~S}_{2}\right)$ : calculated for $\mathrm{C}, 61.19, \mathrm{H}, 3.48$, $\mathrm{N}, 17.84$; found $\mathrm{C}, 61.21, \mathrm{H}, 3.50, \mathrm{~N}, 17.86$.

$\left(5,5^{\prime}\right)$-3,3' -((1)-Indolin-2,3-diylidenebis(azanylylidene))bis(5-nitrobenzylidene)-2-thioxoimidazolidin-4-one (3b): light brown solid; yield: $96 \%$; $\mathrm{mp}: 192^{\circ} \mathrm{C}$; IR $(\mathrm{KBr})$; 3427 (NHstr), 3175 (Ar-CHstr), Ar), 1676 (C=O), 1693 $(\mathrm{C}=\mathrm{Nstr}), 1497(\mathrm{Ar}-\mathrm{C}=\mathrm{C}), 1473\left(\mathrm{NO}_{2}\right.$ str $), 1134(\mathrm{C}=\mathrm{S})\left(\mathrm{cm}^{-}\right.$ $\left.{ }^{1}\right) ;{ }^{1} \mathrm{H}$ NMR $\left(300 \mathrm{MHz}, \mathrm{CDCl}_{3}\right) \delta 12.85$ (s, $2 \mathrm{H}$, imidazolidine-NH), $10.70(\mathrm{~s}, 1 \mathrm{H}$, isatin- $\mathrm{NH}), 8.28(\mathrm{~s}, 2 \mathrm{H}, \mathrm{Ar}-\mathrm{H})$, 8.10 (d, $2 \mathrm{H}, J=7.0 \mathrm{~Hz}, \mathrm{Ar}-\mathrm{H}), 7.73(\mathrm{~d}, 2 \mathrm{H}, J=7.3 \mathrm{~Hz}, \mathrm{Ar}-$ $\mathrm{H}), 7.56(\mathrm{t}, 2 \mathrm{H}, \mathrm{Ar}-\mathrm{H}), 7.50(\mathrm{~d}, 1 \mathrm{H}$, isatin, $J=7.5 \mathrm{~Hz}), 7.32$ $(\mathrm{t}, 1 \mathrm{H}$, isatin, $J=7.7 \mathrm{~Hz}), 7.01(\mathrm{t}, 1 \mathrm{H}$, isatin, $J=7.4 \mathrm{~Hz})$, $6.92(\mathrm{~d}, 1 \mathrm{H}$, isatin, $J=7.8 \mathrm{~Hz}), 6.87(\mathrm{~s}, 2 \mathrm{H}$, imidazolidine $=\mathrm{CH}) ;{ }^{13} \mathrm{C}$ NMR $\left(300 \mathrm{MHz}, \mathrm{CDCl}_{3}\right) \delta 179.08(2 \mathrm{C}, \mathrm{C}-4,7$, $\mathrm{C}=\mathrm{S}), 162.45$ (2C, $\mathrm{C}-2,5, \mathrm{C}=\mathrm{O}), 154.48(2 \mathrm{C}, \mathrm{C}-1,8, \mathrm{C}=\mathrm{N})$, $148.10,137.04,135.96,128.22,121.94,119.34,117.92$ (14C, C-3' $\left.{ }^{\prime}, 6^{\prime}, \mathrm{Ar}\right), 145.72$ (1C, C-14, isatin), 142.05 (2C, C-3,6, imidazolidine- $\mathrm{CH}$ ), 132.78 (1C, C-12, isatin), 131.30 (1C, C-6, isatin), 121.98 (1C, C-13, isatin, Ar), 119.85 (1C, C11, isatin), 110.73 (1C, C-9, isatin); EI-MS (relative intensity $\%): \quad \mathrm{m} / \mathrm{z} \quad 640.08 \quad\left(\mathrm{M}^{+}, \quad 31 \%\right)$. Elemental analysis $\left(\mathrm{C}_{28} \mathrm{H}_{17} \mathrm{~N}_{9} \mathrm{O}_{6} \mathrm{~S}_{2}\right)$ : calculated C, 52.58, H, 2.68, N, 19.71; found $\mathrm{C}, 52.60, \mathrm{H}, 2.71, \mathrm{~N}, 19.73$.

$\left(5,5^{\prime}\right)-3,3^{\prime}-\left(\left(1,1^{\prime}\right)-\right.$ Indoline-2,3-diylidenebis(azanylylidene)bis(5-(2-hydroxybenzylidene)-2-thioxoimidazolidin4-one (3c): brown solid; yield: $97 \%$; $\mathrm{mp}: 226^{\circ} \mathrm{C}$; IR $(\mathrm{KBr})$ 3420 (NHstr), 3264 (OHstr), 3177 (Ar-CHstr), 1674 $(\mathrm{C}=\mathrm{O}), 1695$ (C=Nstr), 1497 (Ar-C=C), $1136(\mathrm{C}=\mathrm{S})\left(\mathrm{cm}^{-1}\right)$; ${ }^{1} \mathrm{H}$ NMR $\left(300 \mathrm{MHz}, \mathrm{CDCl}_{3}\right) \delta 12.53$ (s, $2 \mathrm{H}$, imidazolidine$\mathrm{NH}), 10.63(\mathrm{~s}, 1 \mathrm{H}$, isatin-NH), $9.85(\mathrm{~s}, 2 \mathrm{H}, \mathrm{OH}), 8.22(\mathrm{~d}$, $2 \mathrm{H}, J=7.1 \mathrm{~Hz}, \mathrm{Ar}-\mathrm{H}), 7.53(\mathrm{~d}, 1 \mathrm{H}$, isatin, $J=7.5 \mathrm{~Hz}), 7.30$ $(\mathrm{t}, 1 \mathrm{H}$, isatin, $J=7.7 \mathrm{~Hz}), 7.25(\mathrm{t}, 2 \mathrm{H}, \mathrm{Ar}-\mathrm{H}), 7.04(\mathrm{t}, 1 \mathrm{H}$, isatin, $J=7.4 \mathrm{~Hz}), 6.94(\mathrm{~d}, 1 \mathrm{H}$, isatin, $J=7.8 \mathrm{~Hz}), 6.83(\mathrm{~s}, 2 \mathrm{H}$, imidazolidine $=\mathrm{CH}), 6.80(\mathrm{t}, 2 \mathrm{H}, \mathrm{Ar}-\mathrm{H}), 6.56(\mathrm{~d}, 2 \mathrm{H}, J=7.4$ $\mathrm{Hz}, \mathrm{Ar}-\mathrm{H}) ;{ }^{13} \mathrm{C}$ NMR $\left(300 \mathrm{MHz}, \mathrm{CDCl}_{3}\right) \delta 179.04(2 \mathrm{C}, \mathrm{C}-$ 4,7, C=S), $162.45(2 \mathrm{C}, \mathrm{C}-2,5, \mathrm{C}=\mathrm{O}), 154.48(2 \mathrm{C}, \mathrm{C}-1,8$, $\mathrm{C}=\mathrm{N}), 145.72$ (1C, C-14, isatin), 142.05 (2C, C-3,6, imidazolidine-CH), 142.00, 130.83, 126.70, 120.29, 119.30,118.85, 

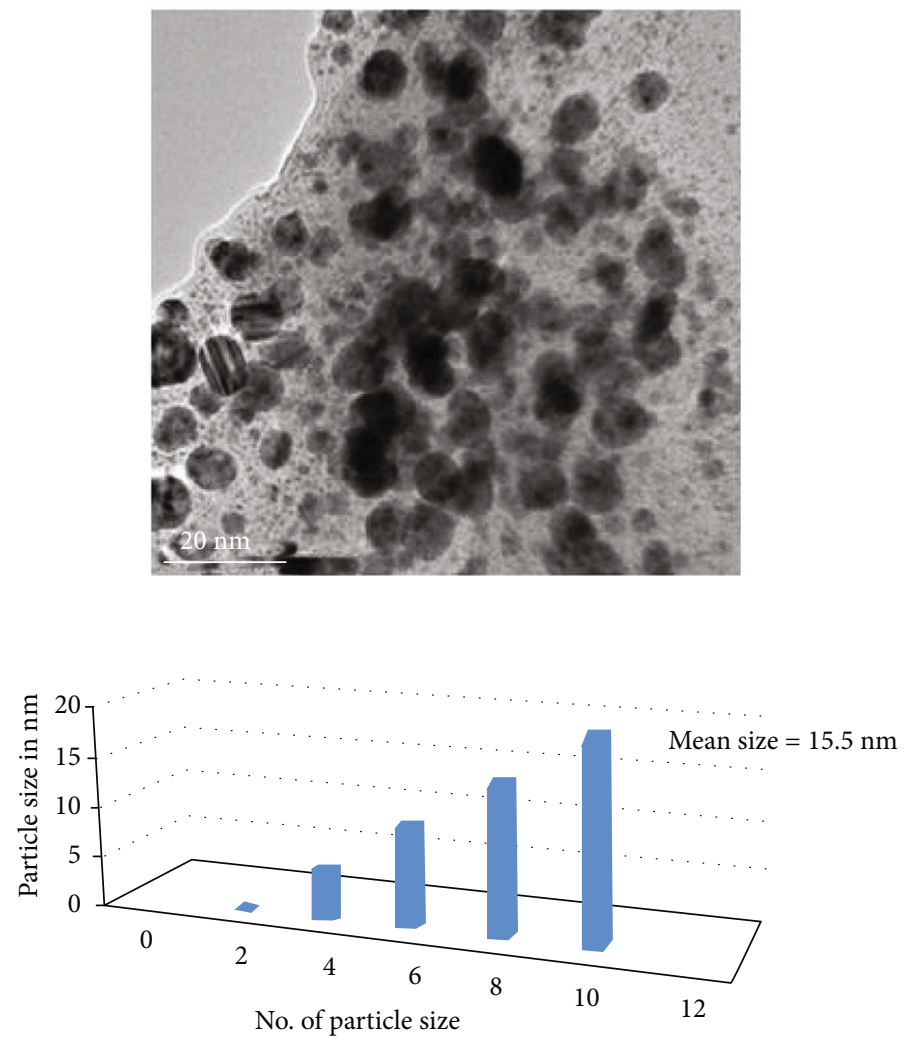

FIGURE 3: TEM image of silver nanoparticle.

116.05 (14C, C-3' $\left.{ }^{\prime} 6^{\prime}, \mathrm{Ar}\right), 132.78$ (1C, C-12, isatin), 131.30 (1C, C-6, isatin), 121.89 (1C, C-13, isatin), 119.61 (1C, C11 , isatin), 110.73 (1C, C-9, isatin); EI-MS (relative intensity $\%): \quad \mathrm{m} / \mathrm{z} \quad 582.10\left(\mathrm{M}^{+}, \quad 31 \%\right)$. Elemental analysis $\left(\mathrm{C}_{28} \mathrm{H}_{19} \mathrm{~N}_{7} \mathrm{O}_{4} \mathrm{~S}_{2}\right)$ : calculated $\mathrm{C}, 57.82, \mathrm{H}, 3.29, \mathrm{~N}, 16.86$; found $\mathrm{C}, 57.80, \mathrm{H}, 3.30, \mathrm{~N}, 16.83$.

$\left(5,5^{\prime}\right)-3,3^{\prime}-((1)$-Indoline-2,3-diylidenebis(azanylylidene)bis(5-(4-hydroxybenzylidene)-2-thioxoimidazolidin-4one (3d): yellow orange solid; yield: $95 \% \mathrm{mp}: 228^{\circ} \mathrm{C}$; IR (KBr) 3426 (NHstr), 3262 (OHstr), 3173 (Ar-CHstr), 1670 (C=O), 1693 (C=Nstr), 1494 (Ar-C=C), $1133(\mathrm{C}=\mathrm{S})\left(\mathrm{cm}^{-1}\right)$; ${ }^{1} \mathrm{H}$ NMR $\left(300 \mathrm{MHz}, \mathrm{CDCl}_{3}\right.$ ), $\delta 12.80$ (s, 2H, imidazolidine$\mathrm{NH}), 10.76(\mathrm{~s}, 1 \mathrm{H}$, isatin-NH), $9.41(\mathrm{~s}, 2 \mathrm{H}, \mathrm{OH}), 7.73$ (d, $4 \mathrm{H}, J=7.6 \mathrm{~Hz}, \mathrm{Ar}-\mathrm{H}), 7.53(\mathrm{~d}, 1 \mathrm{H}$, isatin, $J=7.5 \mathrm{~Hz}), 7.30$ $(\mathrm{t}, 1 \mathrm{H}$, isatin, $J=7.7 \mathrm{~Hz}), 7.02(\mathrm{t}, 1 \mathrm{H}$, isatin, $J=7.4 \mathrm{~Hz})$, $7.01(\mathrm{~d}, 4 \mathrm{H}, J=7.3 \mathrm{~Hz}, \mathrm{Ar}-\mathrm{H}), 6.92(\mathrm{~d}, 1 \mathrm{H}$, isatin, $J=7.8$ $\mathrm{Hz})$, $6.89(\mathrm{~s}, 2 \mathrm{H}$, imidazolidine $=\mathrm{CH}) ;{ }^{13} \mathrm{C} \mathrm{NMR}(300 \mathrm{MHz}$, $\left.\mathrm{CDCl}_{3}\right) \delta 179.05(2 \mathrm{C}, \mathrm{C}-4,7, \mathrm{C}=\mathrm{S}), 162.43$ (2C, C-2,5, $\mathrm{C}=\mathrm{O}), 156.48$ (2C, $\mathrm{C}-1,8, \mathrm{C}=\mathrm{N}), 145.72$ (1C, C-14, isatin), 142.05 (2C, C-3,6, imidazolidine-CH), 142.03, 130.81, 121.98, 119.31, 110.71 (14C, C-3',6', Ar), 132.25 (1C, C12 , isatin), 131.36(1C, C-6, isatin), 121.89 (1C, C-13, isatin), 119.61 (1C, C-11, isatin), 110.73 (1C, C-9, isatin); EI-MS (relative intensity\%): $\mathrm{m} / \mathrm{z} 582.10\left(\mathrm{M}^{+}, 31 \%\right)$. Elemental analysis $\left(\mathrm{C}_{28} \mathrm{H}_{19} \mathrm{~N}_{7} \mathrm{O}_{4} \mathrm{~S}_{2}\right)$ : calculated $\mathrm{C}, 57.82, \mathrm{H}, 3.29, \mathrm{~N}, 16.86$; found $\mathrm{C}, 57.84, \mathrm{H}, 3.32, \mathrm{~N}, 16.88$.

$\left(5,5^{\prime}\right)-3,3^{\prime}-\left(\left(1,1^{\prime}\right)\right.$-Indoline-2,3-diylidenebis(azanylylidene)bis(5-(4-chlorobenzylidene)-2-thioxoimidazolidin-4one (3e): dark yellow solid; yield: $98 \% \mathrm{mp}$ : $230^{\circ} \mathrm{C}$; IR ( $\mathrm{KBr}$ )

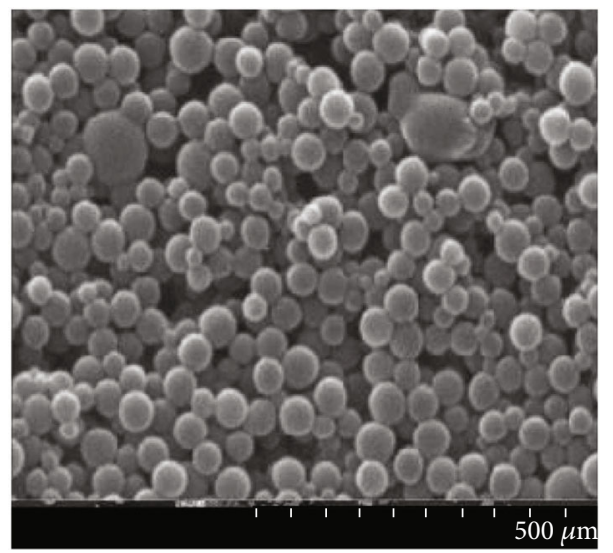

FIgure 4: SEM image of biosynthesized AgNPs.

3420 (NHstr), 3173 (Ar-CHstr), $1670 \quad$ (C=O), 1693 (C=Nstr), 1494 (Ar-C=C), $1133(\mathrm{C}=\mathrm{S}), 759(\mathrm{C}-\mathrm{Cl})\left(\mathrm{cm}^{-1}\right)$; ${ }^{1} \mathrm{H}$ NMR $\left(300 \mathrm{MHz}, \mathrm{CDCl}_{3}\right) \delta 12.78$ (s, 2H, imidazolidine$\mathrm{NH}), 10.73(\mathrm{~s}, 1 \mathrm{H}$, isatin-NH), $7.54(\mathrm{~d}, 1 \mathrm{H}$, isatin, $J=7.5$ $\mathrm{Hz}$ ), $7.50(\mathrm{~d}, 4 \mathrm{H}, J=7.2 \mathrm{~Hz}, \mathrm{Ar}-\mathrm{H}), 7.34(\mathrm{t}, 1 \mathrm{H}$, isatin, $J=$ $7.7 \mathrm{~Hz}), 7.32(\mathrm{~d}, 4 \mathrm{H}, J=7.1 \mathrm{~Hz}, \mathrm{Ar}-\mathrm{H}), 7.06(\mathrm{t}, 1 \mathrm{H}$, isatin, $=7.4 \mathrm{~Hz}), 6.96(\mathrm{~d}, 1 \mathrm{H}$, isatin, $J=7.8 \mathrm{~Hz}), 6.83(\mathrm{~s}, 2 \mathrm{H}$, imidazolidine $=\mathrm{CH}) ;{ }^{13} \mathrm{C} \mathrm{NMR}\left(300 \mathrm{MHz}, \mathrm{CDCl}_{3}\right) \delta 179.08(2 \mathrm{C}$, C-4,7, C=S), 162.45 (2C, C-2,5, C=O), 156.43 (2C, C-1,8, $\mathrm{C}=\mathrm{N}), 145.70$ (1C, C-14, isatin), 142.40, 130.81, 128.45, 128.12, 119.25 (14C, C3' $\left.{ }^{\prime}{ }^{\prime}, \mathrm{Ar}\right), 142.03$ (2C, C-3,6, imidazolidine-CH), 132.23 (1C, C-12, isatin), 131.34 (1C, C-6, 

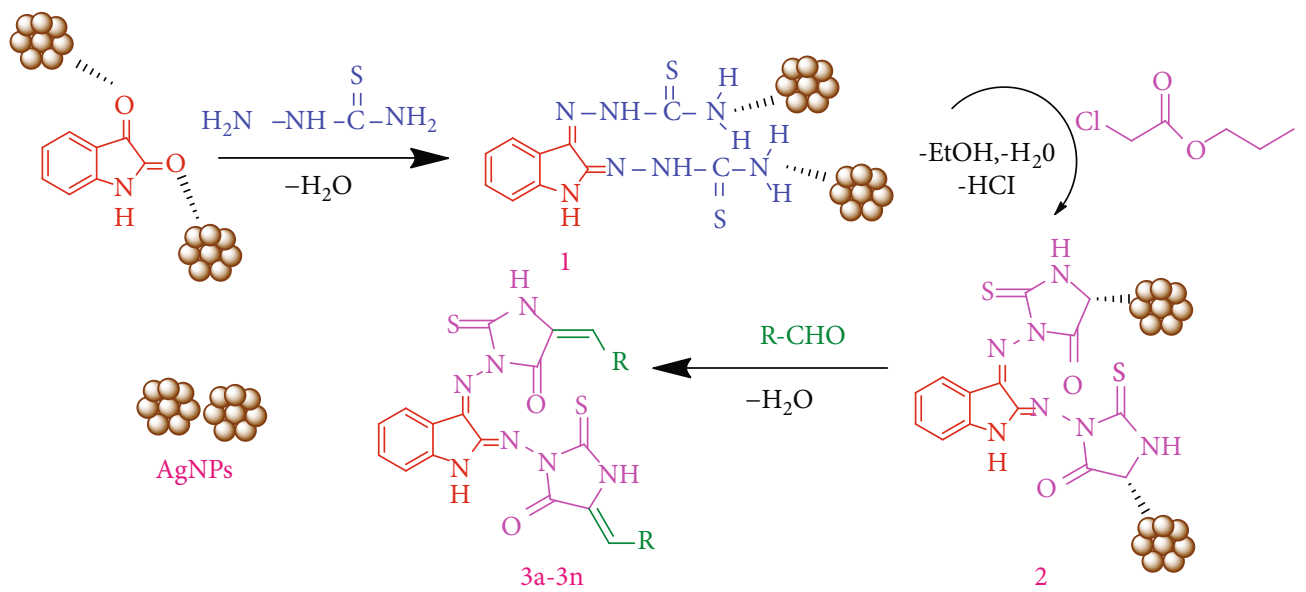

Scheme 2: Synthesis of isatin derivatives. Reagents and conditions: (1) $\mathrm{NH}_{2} \mathrm{NHC}=\mathrm{S}-\mathrm{NH}_{2}, \mathrm{EtOH}$, and $\mathrm{AgNPs}$; (2) $\mathrm{CH} \mathrm{ClCOOEt}_{2}$ $\mathrm{CH}_{3} \mathrm{COONa}, \mathrm{EtOH}$, and AgNPs; (3a-3n) RCHO, EtOH, and AgNPs.

isatin), 121.85 (1C, C-13, isatin), 119.60 (1C, C-11, isatin), 110.73 (1C, C-9, isatin); EI-MS (relative intensity \%): $\mathrm{m} / \mathrm{z}$ $618.03\left(\mathrm{M}^{+}, 32.2 \%\right)$. Elemental analysis $\left(\mathrm{C}_{28} \mathrm{H}_{17} \mathrm{Cl}_{2} \mathrm{~N}_{7} \mathrm{O}_{2} \mathrm{~S}_{2}\right)$ : calculated C, 54.37, H, 2.77, N, 15.85; found C, 54.35, H, 2.75, N, 15.88 .

5-(4-Methoxybenzylidene)-3-(-4-(4-methxoybenzylidene)-5-oxo-2-thioxoimidazolidin-1-yl)imnio)indolin-2-ylidene)amino)-2-thioxoimidazolidin-4-one (3f): dark yellow solid; yield: $94 \% \mathrm{mp}: 234^{\circ} \mathrm{C}$; IR (KBr) 3420 (NHstr), 3173 (Ar-CHstr), 1670 (C=O), 1693 (C=Nstr), 1494 (Ar-C=C), $1133(\mathrm{C}=\mathrm{S})\left(\mathrm{cm}^{-1}\right) ;{ }^{1} \mathrm{H}$ NMR $\left(300 \mathrm{MHz}, \mathrm{CDCl}_{3}\right) \delta 12.73(\mathrm{~s}$, $2 \mathrm{H}$, imidazolidine- $\mathrm{NH}), 10.75(\mathrm{~s}, 1 \mathrm{H}$, isatin- $\mathrm{NH}), 7.88$ (d, $4 \mathrm{H}, J=7.3 \mathrm{~Hz}, \mathrm{Ar}-\mathrm{H}), 7.56(\mathrm{~d}, 1 \mathrm{H}$, isatin, $J=7.5 \mathrm{~Hz}), 7.37$ $(\mathrm{t}, 1 \mathrm{H}$, isatin, $J=7.7 \mathrm{~Hz}), 7.08(\mathrm{t}, 1 \mathrm{H}$, isatin, $J=7.4 \mathrm{~Hz})$, $7.03(\mathrm{~d}, 4 \mathrm{H}, J=7.1 \mathrm{~Hz}, \operatorname{Ar}-\mathrm{H}), 6.94(\mathrm{~d}, 1 \mathrm{H}$, isatin, $J=7.8$ $\mathrm{Hz}$ ), 6.85 (s, 2H, imidazolidine $=\mathrm{CH}), 3.80\left(\mathrm{~s}, 6 \mathrm{H}, \mathrm{OCH}_{3}\right)$; ${ }^{13} \mathrm{C} \mathrm{NMR}\left(300 \mathrm{MHz}, \mathrm{CDCl}_{3}\right) \delta 179.04$ (2C, C-4,7, C=S), 162.43 (2C, C-2,5, C=O), 157.90, 132.22, 127.80, 119.25, 114.42, 55.81 (16C- C-3' $\left.6^{\prime}, \mathrm{Ar}\right), 156.46(2 \mathrm{C}, \mathrm{C}-1,8, \mathrm{C}=\mathrm{N})$, 145.73 (1C, C-14, isatin), 142.06 (2C, C-3,6, imidazolidine$\mathrm{CH}), 132.25$ (1C, C-12, isatin), 131.32 (1C, C-6, isatin), 121.83 (1C, C-13, isatin), 119.60 (1C, C-11, isatin), 110.71 (1C, C-9, isatin); EI-MS (relative intensity \%): $\mathrm{m} / \mathrm{z}$ $610.13\left(\mathrm{M}^{+}, 33 \%\right)$. Elemental analysis $\left(\mathrm{C}_{30} \mathrm{H}_{23} \mathrm{~N}_{7} \mathrm{O}_{4} \mathrm{~S}_{2}\right)$ : calculated C, 59.10, H, 3.80, N, 16.08; found C, 59.12, H, 3.82, $\mathrm{N}, 16.10$.

$\left(5,5^{\prime}\right)-3,3^{\prime}-\left(\left(1,1^{\prime}\right)\right.$-Indoline-2,3-diylidenebis(azanylylidene))bis(5-(3-phenylallylidene)-2-thioxoimidazolidin-4one $(3 \mathrm{~g})$ : light brown solid; yield: $90 \% \mathrm{mp}: 226^{\circ} \mathrm{C}$; IR ( $\left.\mathrm{KBr}\right)$ 3423 (NHstr), 3170 (Ar-CHstr), 1672 (C=O), 1690 (C=Nstr), $1492\left(\right.$ Ar-C=C), $1130(\mathrm{C}=\mathrm{S})\left(\mathrm{cm}^{-1}\right) ;{ }^{1} \mathrm{H}$ NMR $\left(300 \mathrm{MHz}, \mathrm{CDCl}_{3}\right) \delta 12.75(\mathrm{~s}, 2 \mathrm{H}$, imidazolidine- $\mathrm{NH})$, $10.74(\mathrm{~s}, 1 \mathrm{H}$, isatin-NH), $7.60(\mathrm{~d}, 4 \mathrm{H}, J=7.3 \mathrm{~Hz}, \mathrm{Ar}-\mathrm{H})$, $7.56(\mathrm{~d}, 1 \mathrm{H}$, isatin, $J=7.5 \mathrm{~Hz}), 7.37(\mathrm{t}, 1 \mathrm{H}$, isatin, $J=7.7$ $\mathrm{Hz}), 7.35$ (m, 4H, Ar-H), $7.30(\mathrm{~m}, 2 \mathrm{H}, \mathrm{Ar}), 7.05$ (d, 4H, Ar), $7.04(\mathrm{t}, 1 \mathrm{H}$, isatin, $J=7.4 \mathrm{~Hz}), 6.93(\mathrm{~d}, 1 \mathrm{H}$, isatin, $J=$ $7.8 \mathrm{~Hz}), \quad 6.86(\mathrm{~s}, \quad 2 \mathrm{H}$, imidazolidine $=\mathrm{CH}) ;{ }^{13} \mathrm{C} \quad \mathrm{NMR}$ $\left(300 \mathrm{MHz}, \mathrm{CDCl}_{3}\right) \delta 179.06(2 \mathrm{C}, \mathrm{C}-4,7, \mathrm{C}=\mathrm{S}), 162.40(2 \mathrm{C}$, $\mathrm{C}-2,5, \mathrm{C}=\mathrm{O}), 156.42(2 \mathrm{C}, \mathrm{C}-1,8, \mathrm{C}=\mathrm{N}), 145.76$ (1C, C-14,
TABle 1: Optimization of substituent for compounds $3 a-3 n$.

\begin{tabular}{lccc}
\hline Compounds & $R$ & Time (hrs) & Yield (\%) \\
\hline $3 \mathrm{a}$ & $\mathrm{PhCHO}$ & 3 & 90 \\
$3 \mathrm{~b}$ & $3-\mathrm{NO}_{2} \mathrm{PhCHO}$ & 3 & 96 \\
$3 \mathrm{c}$ & $2-\mathrm{OHPhCHO}$ & 3 & 97 \\
$3 \mathrm{~d}$ & $4-\mathrm{OHPhCHO}$ & 3 & 95 \\
$3 \mathrm{e}$ & $4-\mathrm{ClPhCHO}$ & 3 & 98 \\
$3 \mathrm{f}$ & $4-\mathrm{OCH}{ }_{3} \mathrm{PhCHO}$ & 3 & 94 \\
$3 \mathrm{~g}$ & $\mathrm{CH}=\mathrm{CH}^{-} \mathrm{PhCHO}$ & 3 & 90 \\
$3 \mathrm{~h}$ & $\mathrm{Citral}$ & 3 & 93 \\
$3 \mathrm{i}$ & $4-\mathrm{N}\left(\mathrm{CH}_{3}\right)_{2} \mathrm{PhCHO}$ & 3 & 91 \\
$3 \mathrm{j}$ & Vanillin & 3 & 98 \\
$3 \mathrm{k}$ & Furfural & 3 & 91 \\
$3 \mathrm{l}$ & Crotanal & 3 & 89 \\
$3 \mathrm{~m}$ & Glutral & 3 & 92 \\
$3 \mathrm{n}$ & HCHO & 3 & 87 \\
\hline
\end{tabular}

isatin), $\quad 142.03$ (2C, C-3,6, imidazolidine-CH),141.02, $137.62,130.32,130.25,126.43,125.26,119.26$ (18C, C-3', $6^{\prime}$ , Ar), 132.25 (1C, C-12, isatin), 131.35 (1C, C-6, isatin), 121.81 (1C, C-13, isatin), 119.60 (1C, C-11, isatin), 110.72 (1C, C-9, isatin); EI-MS (relative intensity \%): $\mathrm{m} / \mathrm{z} 602.14$ $\left(\mathrm{M}^{+}, 35 \%\right)$. Elemental analysis $\left(\mathrm{C}_{32} \mathrm{H}_{23} \mathrm{~N}_{7} \mathrm{O}_{2} \mathrm{~S}_{2}\right)$ : calculated C, 63.88, H, 3.85, N, 16.29; found C, 63.86, H, 3.83, N, 16.31 .

$\left(5,5^{\prime}\right)-3,3^{\prime}-\left(\left(1,1^{\prime}\right)\right.$-Indoline-2,3-diylidenebis(azanylylidene))bis(5-(-3,7-dimethylocta-2,6-dien-1-ylidene)-2-thioxoimidazolidin-4-one (3h): light yellowish brown solid; yield: 93\% mp: $230^{\circ} \mathrm{C}$; IR (KBr) 3426 (NHstr), 3174 (Ar-CHstr), $1674(\mathrm{C}=\mathrm{O}), 1692$ (C=Nstr), 1494 (Ar-C=C), $1135(\mathrm{C}=\mathrm{S})$ $\left(\mathrm{cm}^{-1}\right) ;{ }^{1} \mathrm{H}$ NMR $\left(300 \mathrm{MHz}, \mathrm{CDCl}_{3}\right) \delta 12.73$ (s, 2H, imidazolidine- $\mathrm{NH}), 10.72(\mathrm{~s}, 1 \mathrm{H}$, isatin- $\mathrm{NH}), 7.52(\mathrm{~d}, 1 \mathrm{H}$, isatin, $J$ $=7.5 \mathrm{~Hz}), 7.37(\mathrm{t}, 1 \mathrm{H}$, isatin, $J=7.7 \mathrm{~Hz}), 7.06(\mathrm{t}, 1 \mathrm{H}$, isatin, $J=7.4 \mathrm{~Hz}), 6.99(\mathrm{~d}, 1 \mathrm{H}$, isatin, $J=7.8 \mathrm{~Hz}), 6.90(\mathrm{~s}, 2 \mathrm{H}$, imidazolidine $=\mathrm{CH}), 6.45,5.15,2.01,1.85(30 \mathrm{H}, \mathrm{m}$, citral $) ;{ }^{13} \mathrm{C}$ NMR $\left(300 \mathrm{MHz}, \mathrm{CDCl}_{3}\right) \delta 177.04$ (2C, C-4,7, C=S), 162.33 


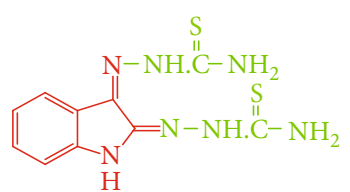

1

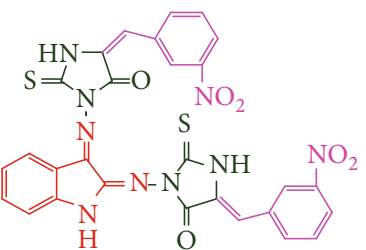

$3 b$<smiles>O=C1N=NC(=S)N/C1=C/c1ccc(Cl)cc1</smiles><smiles>N=C1C(=NN2C(=O)/C(=C/c3ccc(Cl)cc3)NC2=S)Nc2ccccc21</smiles>

$3 e$<smiles>CC(C)=CCCC(C)=CC=C1NC(=S)NC1=O</smiles><smiles>CC(C)=CCC/C(C)=C/C=C1\NC(=S)N(N=C2Nc3ccccc3C2=N)C1=O</smiles>

$3 \mathrm{~h}$

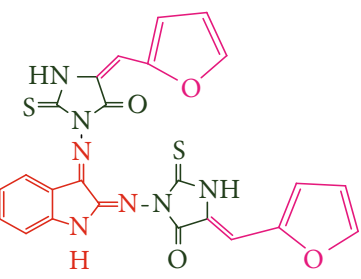

$3 \mathrm{k}$

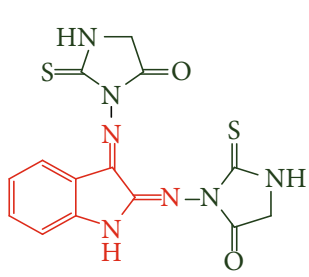

2

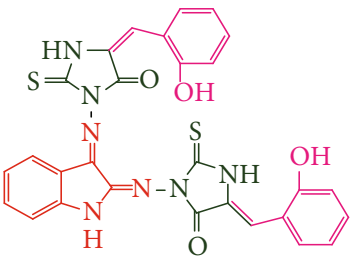

$3 c$

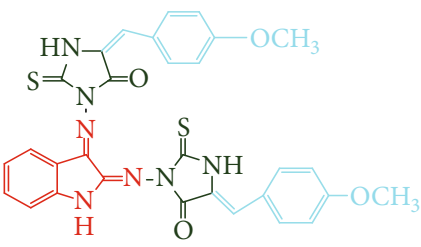

$3 f$
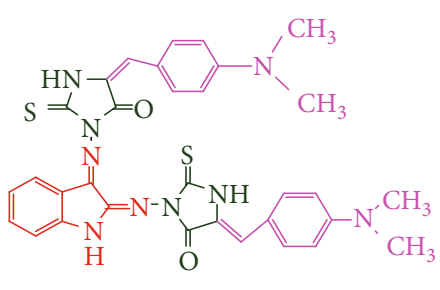

$3 \mathrm{i}$

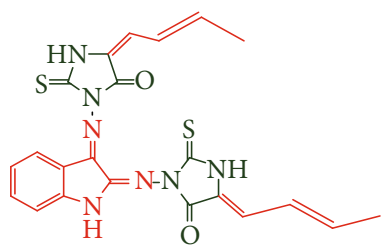

31

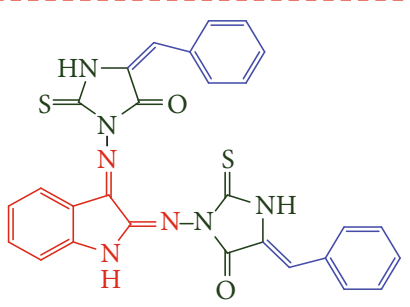

$3 a$

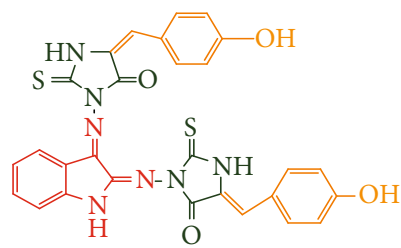

$3 d$

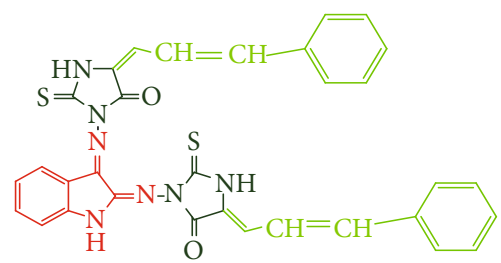

$3 g$

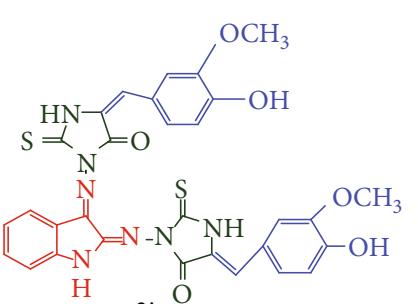

3j

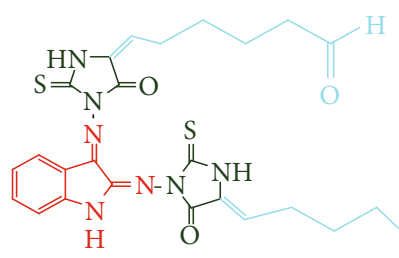

$3 \mathrm{~m}$

$$
\text { (n) }
$$

$3 n$

FIGURE 5: Structure of newly synthesized compounds of isatin-connected thioxoimidazolidine derivatives 1,2 , and $3 \mathrm{a}-3 \mathrm{n}$. 
TABLE 2: Cytotoxic activity screening of compounds (1, 2, and 3a-3n) against breast cancer cell line (MCF-7) compound in various concentrations $(\mu \mathrm{M})$.

\begin{tabular}{|c|c|c|c|c|c|}
\hline Cpd. no. & $10(\mu \mathrm{M})$ & $25(\mu \mathrm{M})$ & $50(\mu \mathrm{M})$ & $100(\mu \mathrm{M})$ & $\mathrm{IC}_{50}(\mu \mathrm{M})$ \\
\hline 1 & $0.892 \pm 0.013$ & $0.724 \pm 0.032$ & $0.534 \pm 0.042$ & $0.621 \pm 0.024$ & 64.2 \\
\hline 2 & $0.996 \pm 0.002$ & $0.526 \pm 0.008$ & $0.523 \pm 0.050$ & $0.542 \pm 0.030$ & 50.0 \\
\hline $3 a$ & $0.954 \pm 0.089$ & $0.654 \pm 0.042$ & $0.472 \pm 0.024$ & $0.441 \pm 0.010$ & 55.1 \\
\hline $3 b$ & $0.910 \pm 0.048$ & $0.698 \pm 0.072$ & $0.460 \pm 0.020$ & $0.602 \pm 0.051$ & 43.4 \\
\hline $3 c$ & $0.810 \pm 0.056$ & $0.435 \pm 0.005$ & $0.452 \pm 0.007$ & $0.482 \pm 0.015$ & 30.1 \\
\hline $3 d$ & $0.657 \pm 0.065$ & $0.458 \pm 0.001$ & $0.533 \pm 0.052$ & $0.523 \pm 0.022$ & 25.3 \\
\hline $3 e$ & $0.560 \pm 0.073$ & $0.394 \pm 0.007$ & $0.421 \pm 0.031$ & $0.450 \pm 0.001$ & 20.5 \\
\hline $3 f$ & $0.974 \pm 0.031$ & $0.679 \pm 0.114$ & $0.563 \pm 0.037$ & $0.854 \pm 0.061$ & 40.7 \\
\hline $3 g$ & $1.786 \pm 0.021$ & $0.690 \pm 0.062$ & $0.551 \pm 0.042$ & $0.412 \pm 0.006$ & 42.8 \\
\hline $3 \mathrm{~h}$ & $0.925 \pm 0.034$ & $0.874 \pm 0.345$ & $0.526 \pm 0.003$ & $0.535 \pm 0.019$ & 44.6 \\
\hline $3 \mathrm{i}$ & $1.870 \pm 0.025$ & $0.956 \pm 0.321$ & $0.751 \pm 0.070$ & $0.610 \pm 0.007$ & 38.7 \\
\hline $3 j$ & $0.923 \pm 0.003$ & $0.887 \pm 0.025$ & $0.819 \pm 0.301$ & $0.646 \pm 0.036$ & 46.0 \\
\hline $3 \mathrm{k}$ & $0.957 \pm 0.348$ & $0.609 \pm 0.013$ & $0.954 \pm 0.004$ & $0.480 \pm 0.022$ & 52.3 \\
\hline 31 & $0.920 \pm 0.049$ & $0.730 \pm 0.125$ & $0.835 \pm 0.060$ & $0.672 \pm 0.035$ & 45.9 \\
\hline $3 \mathrm{~m}$ & $0.963 \pm 0.002$ & $0.593 \pm 0.070$ & $0.856 \pm 0.025$ & $0.468 \pm 0.032$ & 43.7 \\
\hline $3 n$ & $0.895 \pm 0.008$ & $0.882 \pm 0.054$ & $0.760 \pm 0.046$ & $0.473 \pm 0.045$ & 39.0 \\
\hline DOX & $0.801 \pm 0.054$ & $0.586 \pm 0.034$ & $0.670 \pm 0.023$ & $0.768 \pm 0.043$ & 30.10 \\
\hline
\end{tabular}

NA: compound having $\mathrm{IC}_{50}>\mu \mathrm{M}$; each value is the mean of three experiments \pm standard error.

(2C, C-2,5, C=O), $156.23(2 \mathrm{C}, \mathrm{C}-1,8, \mathrm{C}=\mathrm{N}), 145.68$ (1C, C14, isatin), 142.10 (2C, C-3,6, imidazolidine-CH), 140.01, $132.05,125.15,117.20,40.05,26.50,24.50,19.30,18.45$ (20C, C- $3^{\prime}, 6^{\prime}$, citral), 132.15 (1C, C-12, isatin), 131.20 (1C, C-6, isatin), 121.80 (1C, C-13, isatin), 119.62 (1C, C-11, isatin), 110.73 (1C, C-9, isatin); EI-MS (relative intensity \%): $\mathrm{m} / \mathrm{z} 642.26\left(\mathrm{M}^{+}\right.$, $41 \%)$. Elemental analysis $\left(\mathrm{C}_{34} \mathrm{H}_{39} \mathrm{~N}_{7} \mathrm{O}_{2} \mathrm{~S}_{2}\right)$ : calculated C, 63.62, $\mathrm{H}, 6.12, \mathrm{~N}, 15.28$; found $\mathrm{C}, 63.64, \mathrm{H}, 6.15, \mathrm{~N}, 15.30$.

$\left(5,5^{\prime}\right)-3,3^{\prime}-\left(\left(1,1^{\prime}\right)\right.$-Indoline-2,3-diylidenebis(azanylylidene))bis(5-(4-dimethylamino) benzylidene)-2-thioxoimidazolidin-4-one (3i): dark brown solid: yield; 91\% mp: $226^{\circ} \mathrm{C}$; IR (KBr) 3425 (NHstr), 3171 (Ar-CHstr), 1673 $(\mathrm{C}=\mathrm{O}), 1694$ (C=Nstr), 1490 (Ar-C=C), $1137(\mathrm{C}=\mathrm{S})\left(\mathrm{cm}^{-1}\right)$; ${ }^{1} \mathrm{H}$ NMR $\left(300 \mathrm{MHz}, \mathrm{CDCl}_{3}\right) \delta 12.71(\mathrm{~s}, 2 \mathrm{H}$, imidazolidine$\mathrm{NH}), 10.73$ (s, 1H, isatin-NH), 7.63 (d, 4H, J=9.1 Hz, p-aminophenyl), $7.52(\mathrm{~d}, 1 \mathrm{H}$, isatin, $J=7.5 \mathrm{~Hz}), 7.33(\mathrm{t}, 1 \mathrm{H}$, isatin, $J=7.7 \mathrm{~Hz}), 7.04(\mathrm{t}, 1 \mathrm{H}$, isatin, $J=7.4 \mathrm{~Hz}), 6.97(\mathrm{~d}, 1 \mathrm{H}$, isatin, $J=7.8 \mathrm{~Hz}), 6.93(\mathrm{~s}, 2 \mathrm{H}$, imidazolidine $=\mathrm{CH}), 6.75(\mathrm{~d}, 4 \mathrm{H}, J$ $=9.2 \mathrm{~Hz}$, p-aminophenyl $), 4.03\left(\mathrm{~s}, 12 \mathrm{H}, \mathrm{N}\left(\mathrm{CH}_{3}\right)_{2}\right) ;{ }^{13} \mathrm{C}$ NMR (300 MHz, $\mathrm{CDCl}_{3}$ ) $\delta 177.04$ (2C, C-4,7, C=S), 162.28 (2C, C-2,5, C=O), 156.30 (2C, C-1,8, C=N), 151.70, 128.90, $120.82,117.23,111.63,20.45$ (18C, C-3 ${ }^{\prime}, 6^{\prime}$, p-aminophenyl), 145.70 (1C, C-14, isatin), 142.11 (2C, C-3,6, imidazolidine$\mathrm{CH}), 132.13$ (1C, C-12, isatin), 131.23 (1C, C-6, isatin), 121.84 (1C, C-13, isatin), 119.64 (1C, C-11, isatin), 110.72 (1C, C-9, isatin); EI-MS (relative intensity \%): $\mathrm{m} / \mathrm{z} 636.19$ $\left(\mathrm{M}^{+}, 40 \%\right)$. Elemental analysis $\left(\mathrm{C}_{32} \mathrm{H}_{29} \mathrm{~N}_{9} \mathrm{O}_{2} \mathrm{~S}_{2}\right)$ : calculated C, 60.45, H, 4.60, N, 19.83; found C, 60.47, H, 4.62, N, 19.80 .

$\left(5,5^{\prime}\right)-3,3^{\prime}-\left(\left(1,1^{\prime}\right)\right.$-Indoline-2,3-diylidenebis(azanylylidene))bis(5-(4-hydroxy-3-methoxy benzylidene)-2-thioxoi-
TABLE 3: In vitro cytotoxicity of isatin derivatives (1, 2, and 3a-3n) on normal cells.

\begin{tabular}{lcc}
\hline Copd. no. & $\begin{array}{c}\text { MCF-10A } \\
\mathrm{IC}_{50}(\mu \mathrm{M})\end{array}$ & $\begin{array}{c}\text { MRC5 } \\
\mathrm{IC}_{50}(\mu \mathrm{M})\end{array}$ \\
\hline 1 & 75.26 & 70.25 \\
2 & 63.10 & 65.87 \\
3a & 70.15 & 73.30 \\
3b & 58.31 & 54.63 \\
3c & 73.40 & 75.45 \\
3d & 77.38 & 80.31 \\
3e & 80.06 & 86.12 \\
3f & 56.34 & 50.08 \\
3g & 59.50 & 54.29 \\
3h & 67.14 & 61.42 \\
3i & 52.18 & 52.84 \\
$3 \mathrm{j}$ & 65.03 & 67.13 \\
$3 \mathrm{k}$ & 83.26 & 56.27 \\
3l & 78.07 & 60.52 \\
3m & 71.39 & 58.76 \\
3n & 63.02 & 79.07 \\
\hline
\end{tabular}

Each compound was tested in triplicate. All error bars represent mean $\pm \mathrm{SD}$ from three independent experiments.

midazolidin-4-one (3j): brown solid; yield: $98 \% \mathrm{mp}: 228^{\circ} \mathrm{C}$; IR (KBr) 3428 (NHstr), 3262 (OHstr), 3175 (Ar-CHstr), $1676(\mathrm{C}=\mathrm{O}), 1695$ (C=Nstr), 1492 (Ar-C=C), $1133(\mathrm{C}=\mathrm{S})$ $\left(\mathrm{cm}^{-1}\right) ;{ }^{1} \mathrm{H}$ NMR $\left(300 \mathrm{MHz}, \mathrm{CDCl}_{3}\right) \delta 12.70(\mathrm{~s}, 2 \mathrm{H}$, imidazolidine-NH), 10.75 (s, 1H, isatin- $\mathrm{NH}), 7.50(\mathrm{~d}, 1 \mathrm{H}$, isatin, $J$ 


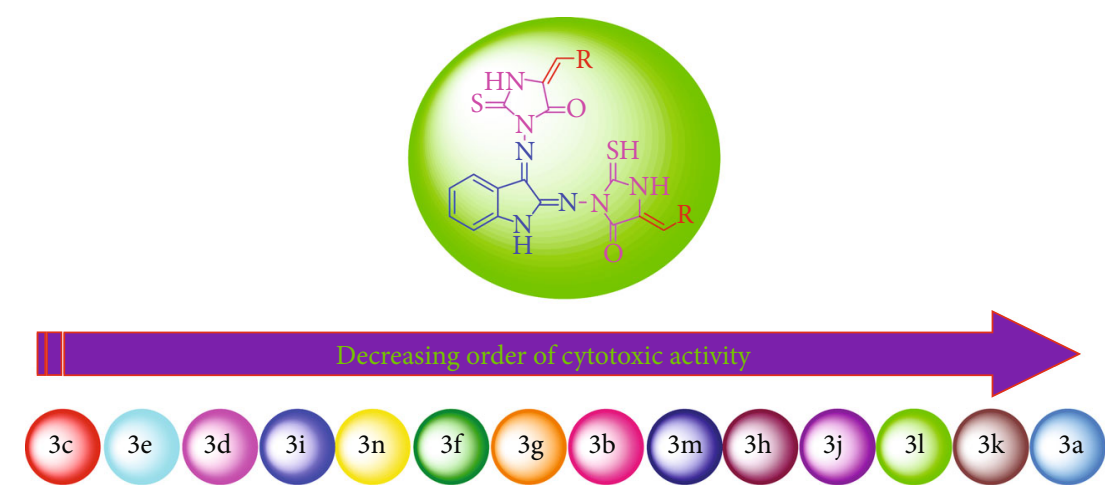

FIgURE 6: The cytotoxic sequence of the synthetic compounds.

$=7.5 \mathrm{~Hz}), 7.35(\mathrm{t}, 1 \mathrm{H}$, isatin, $J=7.7 \mathrm{~Hz}), 7.30(\mathrm{~s}, 2 \mathrm{H}$, vanillin), $7.15(\mathrm{~s}, 2 \mathrm{H}$, vanillin), $7.06(\mathrm{t}, 1 \mathrm{H}$, isatin, $J=7.4 \mathrm{~Hz})$, 7.01 (d, 2H, vanillin), 6.99 (d, $1 \mathrm{H}$, isatin, $J=7.8 \mathrm{~Hz}), 6.90$ $(\mathrm{s}, 2 \mathrm{H}$, imidazolidine $=\mathrm{CH}),(5.38(\mathrm{~s}, 2 \mathrm{H}, \mathrm{OH}), 3.83(\mathrm{~s}, 6 \mathrm{H}$, $\left.\left(\mathrm{OCH}_{3}\right)_{2}\right) ;{ }^{13} \mathrm{C} \mathrm{NMR}\left(300 \mathrm{MHz}, \mathrm{CDCl}_{3}\right) \delta 177.02$ (2C, C4,7, C=S), 162.30 (2C, C-2,5, C=O), 156.25 (2C, C-1,8, $\mathrm{C}=\mathrm{N}), \quad 147.60,146.90,130.50,127.73,123.50,117.20$, 115.60, 56.20 (16C, C-3' $6^{\prime}$, vanillin), 145.68 (1C, C-14, isatin), 142.10 (2C, C-3,6, imidazolidine-CH), 132.15 (1C, C12 , isatin), 131.20 (1C, C-6, isatin), 121.80 (1C, C-13, isatin), 119.62 (1C, C-11, isatin), 110.73 (1C, C-9, isatin); EI-MS (relative intensity \%): $\mathrm{m} / \mathrm{z} 642.12\left(\mathrm{M}^{+}, 33 \%\right)$. Elemental analysis $\left(\mathrm{C}_{30} \mathrm{H}_{23} \mathrm{~N}_{7} \mathrm{O}_{6} \mathrm{~S}_{2}\right)$ : calculated $\mathrm{C}, 56.15, \mathrm{H}, 3.61, \mathrm{~N}$, 15.28; found $\mathrm{C}, 56.18, \mathrm{H}, 3.63, \mathrm{~N}, 15.30$.

$\left(5,5^{\prime}\right)-3,3^{\prime}-\left(\left(1,1^{\prime}\right)\right.$-Indoline-2,3-diylidenebis(azanylylidene))bis(5-(furan-2-ylmethylene)-2-thioxoimidazolidin-4one (3k): yellowish brown solid; yield: $91 \% \mathrm{mp}$ : $240^{\circ} \mathrm{C}$; IR (KBr) 3428 (NHstr), 3173 (Ar-CHstr), 1675 (C=O), 1692 (C=Nstr), 1490 (Ar-C=C), $1139(\mathrm{C}=\mathrm{S})\left(\mathrm{cm}^{-1}\right) ;{ }^{1} \mathrm{H}$ NMR $\left(300 \mathrm{MHz}, \mathrm{CDCl}_{3}\right) \delta 12.73(\mathrm{~s}, 2 \mathrm{H}$, imidazolidine- $\mathrm{NH})$, $10.72(\mathrm{~s}, 1 \mathrm{H}$, isatin-NH), $7.54(\mathrm{~d}, 1 \mathrm{H}$, isatin, $J=7.5 \mathrm{~Hz}$ ),7.53 (d, 2H, furyl ring, $J=3.6 \mathrm{~Hz}), 7.35(\mathrm{t}, 1 \mathrm{H}$, isatin, $J=$ $7.7 \mathrm{~Hz}), 7.02(\mathrm{t}, 1 \mathrm{H}$, isatin, $J=7.4 \mathrm{~Hz}), 6.95(\mathrm{~d}, 1 \mathrm{H}$, isatin, $J$ $=7.8 \mathrm{~Hz}), 6.93(\mathrm{~s}, 2 \mathrm{H}$, imidazolidine $=\mathrm{CH}), 6.35(\mathrm{t}, 2 \mathrm{H}$, furyl ring), $6.10(\mathrm{~d}, 2 \mathrm{H}$, furyl ring, $J=3.3 \mathrm{~Hz}) ;{ }^{13} \mathrm{C} \mathrm{NMR}$ $\left(300 \mathrm{MHz}, \mathrm{CDCl}_{3}\right) \delta 179.04(2 \mathrm{C}, \mathrm{C}-4,7, \mathrm{C}=\mathrm{S}), 162.38(2 \mathrm{C}$, $\mathrm{C}-2,5, \mathrm{C}=\mathrm{O}), 156.40$ (2C, C-1,8, C=N), 152.10, 142.60, $117.26,110.85,105.70$ (10C, C-3' ${ }^{\prime} 6^{\prime}$, furyl ring), 145.74 (1C, C-14, isatin), 142.01 (2C, C-3,6, imidazolidine-CH), 132.23 (1C, C-12, isatin), 131.33 (1C, C-6, isatin), 121.84 (1C, C-13, isatin), 119.62 (1C, C-11, isatin), 110.70 (1C, C9, isatin); EI-MS (relative intensity \%): $\mathrm{m} / \mathrm{z} 530.07\left(\mathrm{M}^{+}\right.$, $26 \%)$. Elemental analysis $\left(\mathrm{C}_{24} \mathrm{H}_{15} \mathrm{~N}_{7} \mathrm{O}_{4} \mathrm{~S}_{2}\right)$ : calculated $\mathrm{C}$, 54.43, H, 2.86, N, 18.52; found C, 54.46, H, 2.83, N, 18.54 .

$\left(5,5^{\prime}\right)-3,3^{\prime}-((1)$-Indoline-2,3-diylidenebis(azanylylidene))bis(5-(-but-2-en-1-ylidene)-2-thioxoimidazolidin-4one) (31): light brown solid; yield: $89 \% \mathrm{mp}: 250^{\circ} \mathrm{C}$; IR ( $\left.\mathrm{KBr}\right)$ 3428 (NHstr), 3172 (Ar-CHstr), 1676 (C=O), 1696 (C=Nstr), 1492 (Ar-C=C), $1137(\mathrm{C}=\mathrm{S})\left(\mathrm{cm}^{-1}\right) ;{ }^{1} \mathrm{H}$ NMR $\left(300 \mathrm{MHz}, \mathrm{CDCl}_{3}\right) \delta 12.73(\mathrm{~s}, 2 \mathrm{H}$, imidazolidine- $\mathrm{NH})$, $10.72(\mathrm{~s}, 1 \mathrm{H}$, isatin-NH), $7.52(\mathrm{~d}, 1 \mathrm{H}$, isatin, $J=7.5 \mathrm{~Hz})$, $7.45(\mathrm{~d}, 2 \mathrm{H}, J=7.3 \mathrm{~Hz}$, crotanal), $7.37(\mathrm{t}, 1 \mathrm{H}$, isatin, $J=7.7$ $\mathrm{Hz}), 7.06(\mathrm{t}, 1 \mathrm{H}$, isatin, $J=7.4 \mathrm{~Hz}), 6.99(\mathrm{~d}, 1 \mathrm{H}$, isatin, $J=$
$7.8 \mathrm{~Hz}), 6.90(\mathrm{~s}, 2 \mathrm{H}$, imidazolidine $=\mathrm{CH}), 6.30(\mathrm{~d}, 2 \mathrm{H}, J=$ $7.5 \mathrm{~Hz}$, crotanal), 5.75 (s, 4H, crotanal), $2.10\left(\mathrm{~s}, 6 \mathrm{H}, \mathrm{CH}_{3}\right)$; ${ }^{13} \mathrm{C} \mathrm{NMR}\left(300 \mathrm{MHz}, \mathrm{CDCl}_{3}\right) \delta 177.02$ (2C, C-4,7, C=S), 162.30 (2C, C-2,5, C=O), 156.28 (2C, C-1,8, C=N), 145.65 (1C, C-14, isatin), 142.12 (2C, C-3,6, imidazolidine-CH), $134.45,129.50,117.24,26.63,14.45$ (10C, C-3', $6^{\prime}$, crotanal), 132.12 (1C, C-12, isatin), 131.23 (1C, C-6, isatin), 121.78 (1C, C-13, isatin), 119.60 (1C, C-11, isatin), 110.70 (1C, C9, isatin); EI-MS (relative intensity \%): $\mathrm{m} / \mathrm{z} 478.11\left(\mathrm{M}^{+}\right.$, 24\%). Elemental analysis $\left(\mathrm{C}_{24} \mathrm{H}_{23} \mathrm{~N}_{7} \mathrm{O}_{2} \mathrm{~S}_{2}\right)$ : calculated $\mathrm{C}$, 57.01, H, 4.59, N, 19.39; found C, 57.03, H, 4.61, N, 19.41 .

$(5,5)-5,5^{\prime}-\left(1,1^{\prime}\right)-\left(\left(1,1^{\prime}\right)-\right.$ Indoline-2,3-diylidenebis(azanylylidene))bis(5-oxo-2-thioxoimida zolidin-1-yl-4-ylidnene))dipentanal (3m): light brown solid; yield: $92 \% \mathrm{mp}$ : $244^{\circ} \mathrm{C}$; IR (KBr) 3425 (NHstr), 3170 (Ar-CHstr), 1673 (C=O), 1694 (C=Nstr), 1494 (Ar-C=C), $1133(\mathrm{C}=\mathrm{S})\left(\mathrm{cm}^{-1}\right)$; ${ }^{1} \mathrm{H} \mathrm{NMR}\left(300 \mathrm{MHz}, \mathrm{CDCl}_{3}\right) \delta 12.76$ (s, 2H, imidazolidine$\mathrm{NH}), 10.73(\mathrm{~s}, 1 \mathrm{H}$, isatin-NH), 9.75 (s, 2H, glutral), 7.50 $(\mathrm{d}, 1 \mathrm{H}$, isatin, $J=7.5 \mathrm{~Hz}), 7.37(\mathrm{t}, 1 \mathrm{H}$, isatin, $J=7.7 \mathrm{~Hz})$, $7.04(\mathrm{t}, 1 \mathrm{H}$, isatin, $J=7.4 \mathrm{~Hz}), 6.98(\mathrm{~d}, 1 \mathrm{H}$, isatin, $J=7.8$ $\mathrm{Hz}), 6.93(\mathrm{~s}, 2 \mathrm{H}$, imidazolidine $=\mathrm{CH}), 2.40(\mathrm{~d}, 2 \mathrm{H}, J=1.5$ $\mathrm{Hz}$, glutral), 2.20 (d, $2 \mathrm{H}, J=1.5 \mathrm{~Hz}$, glutral), $1.86(\mathrm{t}, 2 \mathrm{H}$, glutral); ${ }^{13} \mathrm{C} \mathrm{NMR}\left(300 \mathrm{MHz}, \mathrm{CDCl}_{3}\right) \delta 202.05,117.24,43.65$, 24.03, 21.75 (10C, C-3',6', glutral), 177.02 (2C, C-4,7, $\mathrm{C}=\mathrm{S}), 162.30(2 \mathrm{C}, \mathrm{C}-2,5, \mathrm{C}=\mathrm{O}), 156.28(2 \mathrm{C}, \mathrm{C}-1,8, \mathrm{C}=\mathrm{N})$, 145.65 (1C, C-14, isatin), 142.12 (2C, C-3,6, imidazolidine$\mathrm{CH}), 132.12$ (1C, C-12, isatin), 131.23 (1C, C-6, isatin), 121.78 (1C, C-13, isatin), 119.60 (1C, C-11, isatin), 110.70 (1C, C-9, isatin); EI-MS (relative intensity \%): $\mathrm{m} / \mathrm{z} 538.11$ $\left(\mathrm{M}^{+}, 26 \%\right)$. Elemental analysis $\left(\mathrm{C}_{24} \mathrm{H}_{23} \mathrm{~N}_{7} \mathrm{O}_{2} \mathrm{~S}_{2}\right)$ : calculated C, 53.62, H, 4.31, N, 18.24; found C, 53.65, H, 4.33, N, 18.26.

$3,3^{\prime}-\left(\left(1,1^{\prime}\right)\right.$-Indoline-2,3-diylidenebis(azanylylidene))bis(5-methylene-2-thioxoimidazolidin-4-one (3n): light brown solid; yield: $87 \% \mathrm{mp}: 242^{\circ} \mathrm{C}$; IR (KBr) 3423 (NHstr), 3174 (Ar-CHstr), 1676 (C=O), 1694 (C=Nstr), 1492 (Ar$\mathrm{C}=\mathrm{C}), 1130(\mathrm{C}=\mathrm{S})\left(\mathrm{cm}^{-1}\right) ;{ }^{1} \mathrm{H} \mathrm{NMR}\left(300 \mathrm{MHz}, \mathrm{CDCl}_{3}\right) \delta$ $12.72(\mathrm{~s}, 2 \mathrm{H}$, imidazolidine- $\mathrm{NH}), 10.70(\mathrm{~s}, 1 \mathrm{H}$, isatin- $\mathrm{NH})$, $7.50(\mathrm{~d}, 1 \mathrm{H}$, isatin, $J=7.5 \mathrm{~Hz}), 7.37(\mathrm{t}, 1 \mathrm{H}$, isatin, $J=7.7$ $\mathrm{Hz}), 7.04(\mathrm{t}, 1 \mathrm{H}$, isatin, $J=7.4 \mathrm{~Hz}), 6.98(\mathrm{~d}, 1 \mathrm{H}$, isatin, $J=$ $7.8 \mathrm{~Hz}), 6.93(\mathrm{~s}, 2 \mathrm{H}$, imidazolidine $=\mathrm{CH}), 5.80(\mathrm{~s}, 2 \mathrm{H}$, formyl); ${ }^{13} \mathrm{C}$ NMR $\left(300 \mathrm{MHz}, \mathrm{CDCl}_{3}\right) \delta 177.05$ (2C, C-4,7, $\mathrm{C}=\mathrm{S}), 162.35(2 \mathrm{C}, \mathrm{C}-2,5, \mathrm{C}=\mathrm{O}), 156.26(2 \mathrm{C}, \mathrm{C}-1,8, \mathrm{C}=\mathrm{N})$, 145.62 (1C, C-14, isatin), 142.10 (2C, C-3,6, imidazolidine- 


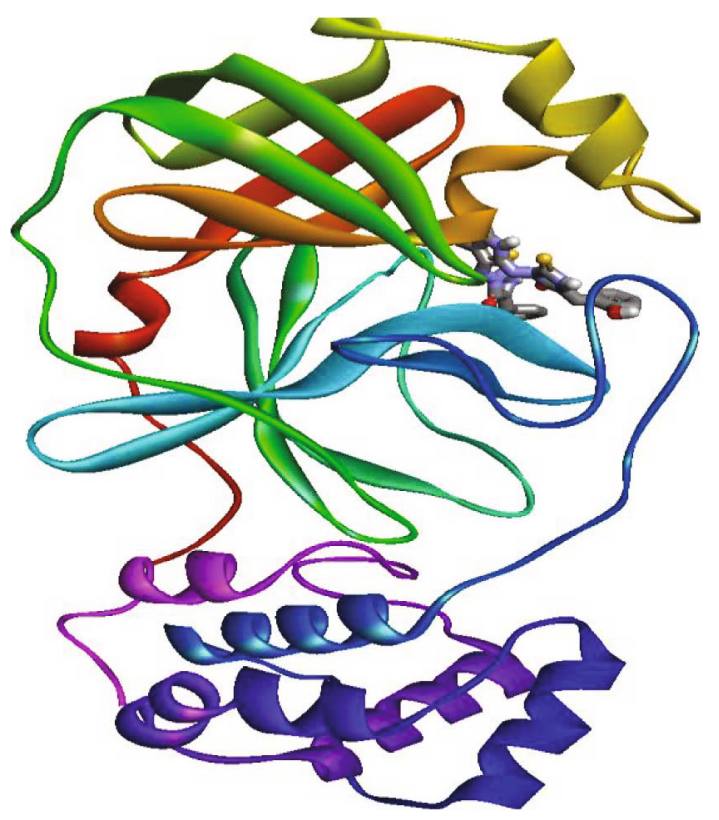

(a)

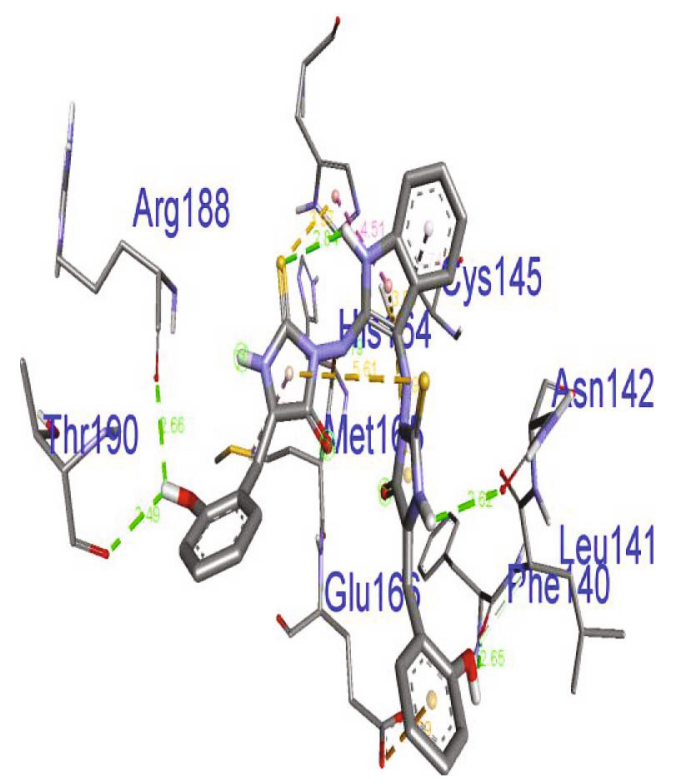

(c)

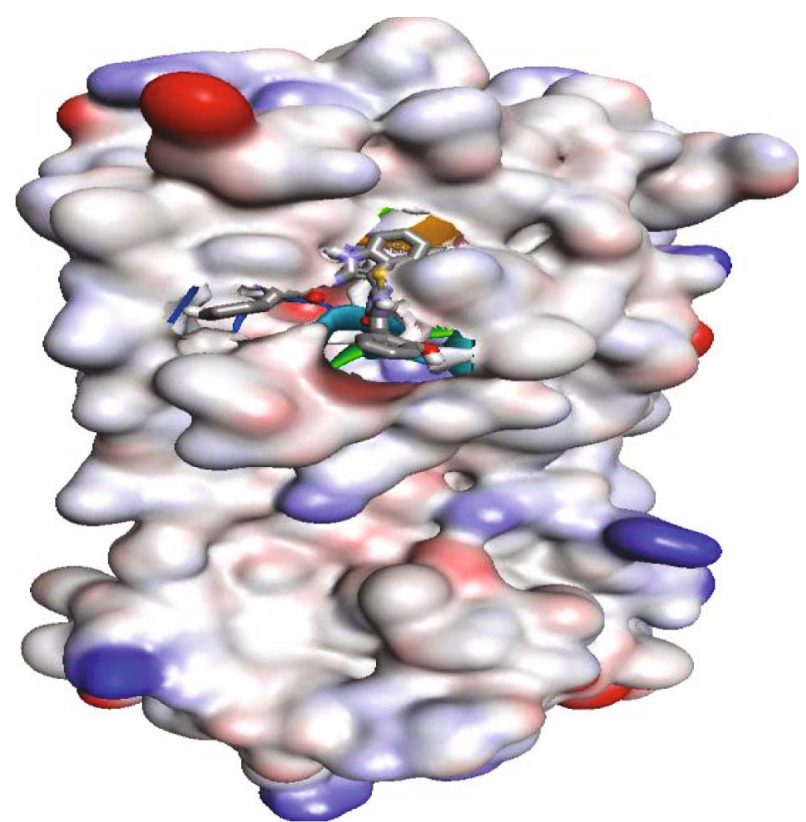

(b)

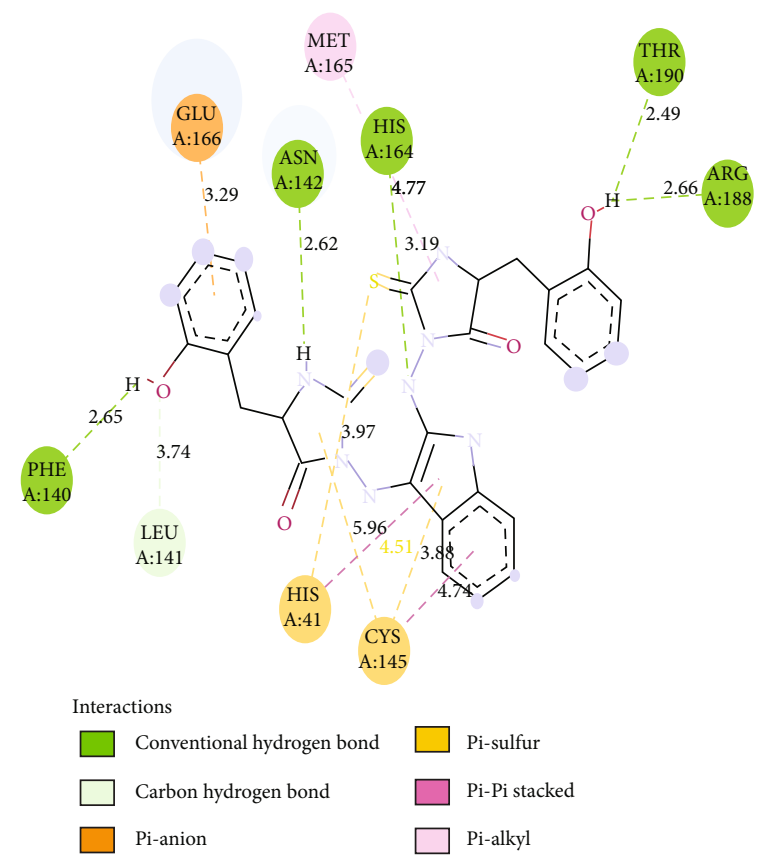

(d)

FIGURE 7: (a) Helix structure of compound 3c; (b) molecular surface of compound 3c; (c) 3D structure of compound 3c; (d) 2D interaction modes of compound $3 c$ within the binding site of 6 LU7 protein.

$\mathrm{CH}), 132.13$ (1C, C-12, isatin), 131.20 (1C, C-6, isatin), 121.76 (1C, C-13, isatin), 119.64 (1C, C-11, isatin), 117.20, 102.40 (4C, C-3' $6^{\prime}$, formyl), 110.68 (1C, C-9, isatin); EIMS (relative intensity \%): $\mathrm{m} / \mathrm{z} 398.04\left(\mathrm{M}^{+}, 21 \%\right)$. Elemental analysis $\left(\mathrm{C}_{16} \mathrm{H}_{11} \mathrm{~N}_{7} \mathrm{O}_{2} \mathrm{~S}_{2}\right)$ : calculated $\mathrm{C}, 48.35, \mathrm{H}, 2.79, \mathrm{~N}$, 24.67; found C, 48.33, H, 2.82, N, 24.65.

2.4. Cytotoxic Activity. Sulforhodamine B stain (SRB) assays were tested for cytotoxic activity of compounds 1, 2, and 3a$3 \mathrm{n}$ using the previous procedure [45]. Cells were plated for
24 hours in a 96-multiwell plate (104 cells/well). To allow cells to adhere to the plate's wall before being treated with the compound(s). Compounds were soluble in DMSO. Different concentrations of the compound under investigation $(10,25,50$, and $100 \mathrm{M}$ ) were injected into the cell monolayer. For each concentration, triplicate wells were prepared. Monolayer cells were incubated for $48 \mathrm{~h}$ at $37^{\circ} \mathrm{C}$ with the compound(s) and $5 \%$ $\mathrm{CO}_{2}$ in the atmosphere. After $48 \mathrm{~h}$, with $0.4 \%(W / V) \mathrm{SRB}$ dissolved in 1 percent acetic acid, the cells were fixed, washed, and stained for $30 \mathrm{~min}$. Four washes with $1 \%$ acetic acid 


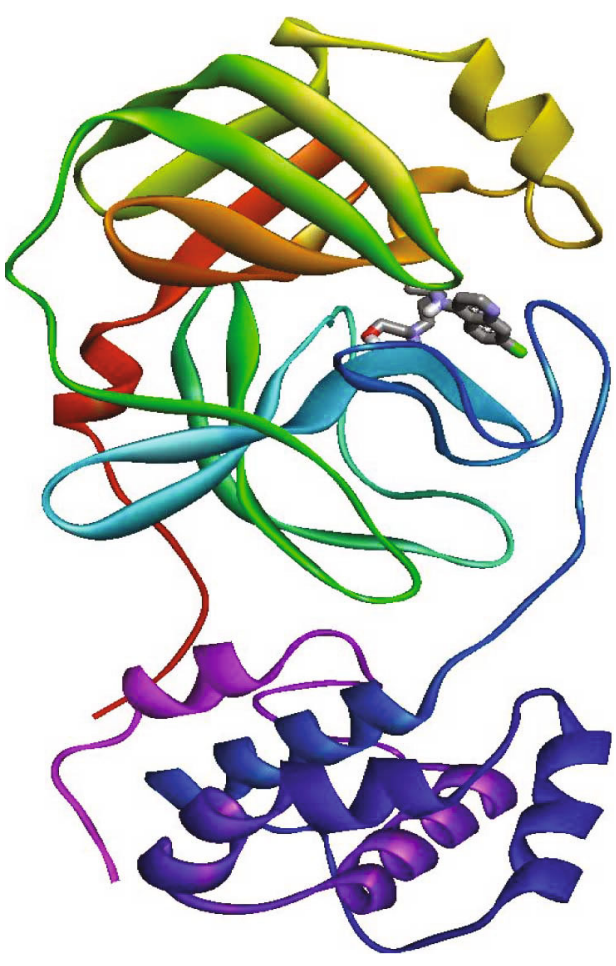

(a)

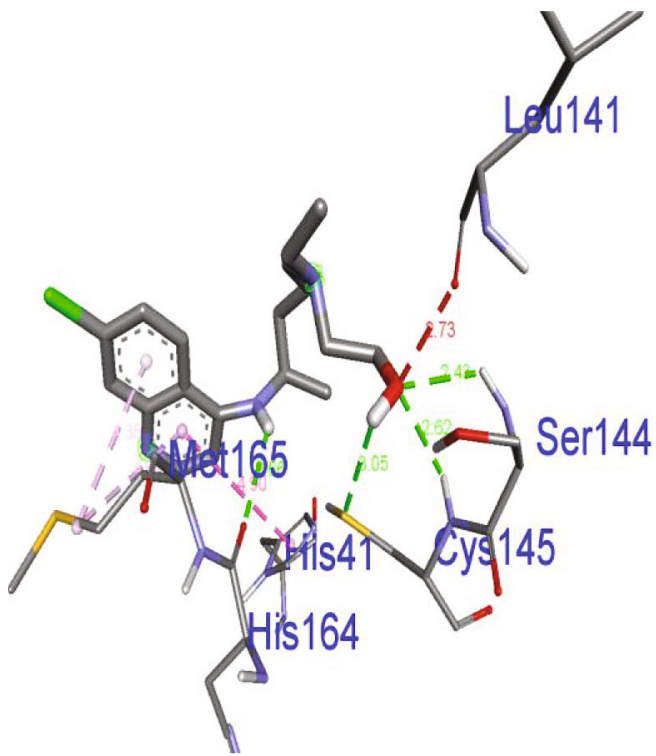

(c)

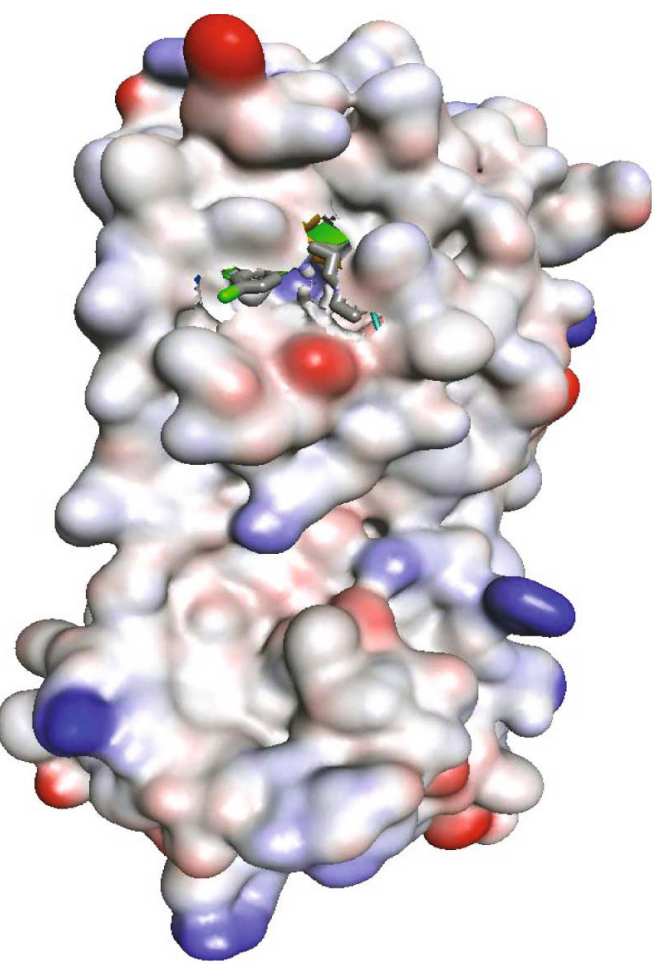

(b)

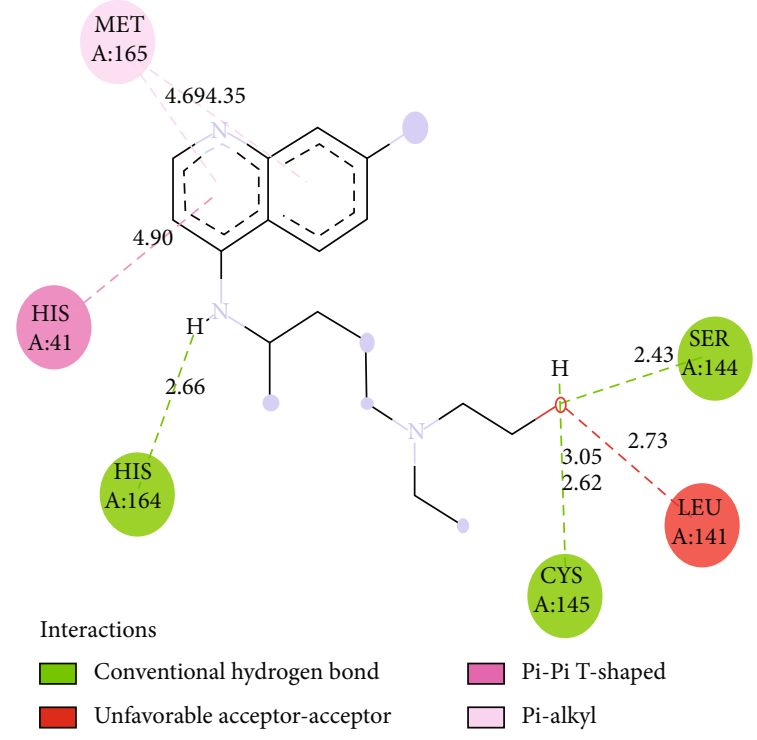

(d)

FIGURE 8: (a) Helix structure of hydroxychloroquine; (b) molecular surface of hydroxychloroquine; (c) 3D structure of hydroxychloroquine; (d) $2 \mathrm{D}$ interaction modes of compound hydroxychloroquine within the binding site of 6LU7 protein.

eliminated the residual unbound dye and the attached stain was recovered with a Tris-EDTA buffer. The strength of the color was calculated by an ELISA reader. Color intensity was measured in an ELISA reader. The molar concentration needed for cell viability inhibition $\left(\mathrm{IC}_{50}\right)$ of $50 \%$ was calculated and compared with the control doxorubicin.

2.5. Molecular Docking Studies. The binding interactions and association of synthetic isatin analogs $1,2,3 a-3 n$, and hydroxychloroquine against target protein (PDB ID: 6LU7) with AutoDock Vina 1.1.2 [46] were investigated using molecular docking studies. The Protein Data Bank (http:// www.rcsb.org) was used to obtain the crystal structure of the SARS-CoV-2 coronavirus core protease (PDB ID: 6LU7). ChemDraw Ultra 12.0 and Chem3D Pro 12.0 software suites were used to sketch the $3 \mathrm{D}$ structures of the ligand molecules and to energy minimization. AutoDock Tools 1.5.6 was used to build the AutoDock Vina input 
TABLE 4: Molecular docking interaction of compounds 1, 2, and 3a-3n against protease of SARS-CoV-2 coronavirus in complex with isatin derivatives (PDB ID: 6LU7).

\begin{tabular}{|c|c|c|c|}
\hline \multirow[t]{2}{*}{ Compounds } & \multicolumn{2}{|c|}{$\begin{array}{l}\text { Main protease of SARS-CoV-2 coronavirus (PDB ID: } \\
\text { 6LU7) }\end{array}$} & \multirow[b]{2}{*}{ H-bonding residues } \\
\hline & Binding affinity $(\mathrm{kcal} / \mathrm{mol})$ & No. of H-bonds & \\
\hline 1 & -6.1 & 5 & His41, Leu141, Cys145, His164 \\
\hline 2 & -7.3 & 3 & Met165, Glu166, Thr190 \\
\hline $3 a$ & -7.8 & 2 & Asn142, Glu166 \\
\hline $3 b$ & -8.9 & 4 & Leu141, Gly143, His164, Glu166 \\
\hline $3 c$ & -9.4 & 5 & Phe140, Asn142, His164, Arg188, Thr190 \\
\hline $3 \mathrm{~d}$ & -8.7 & 2 & Thr190 \\
\hline $3 e$ & -8.2 & 2 & Asn142, His164 \\
\hline $3 \mathrm{f}$ & -8.1 & 1 & His164 \\
\hline $3 g$ & -7.8 & 0 & - \\
\hline $3 \mathrm{~h}$ & -6.2 & 1 & Gly143 \\
\hline $3 \mathrm{i}$ & -7.2 & 3 & Asn142, Glu166 \\
\hline $3 j$ & -7.8 & 1 & Thr26 \\
\hline $3 \mathrm{k}$ & -8.3 & 2 & Cys145, Glu166 \\
\hline 31 & -8.1 & 1 & His164 \\
\hline $3 m$ & -7.2 & 0 & - \\
\hline $3 n$ & -7.9 & 3 & Met165, Glu166, Thr190 \\
\hline Hydroxychloroquine & -6.2 & 3 & Ser144, Cys145, His164 \\
\hline
\end{tabular}

scripts. In build, a cocrystallized inhibitor molecule was used to identify the binding pocket with the discovery studio program. The amino acid residues Thr24, Thr26, Phe140, Asn142, Gly143, Cys145, His163, His164, Glu166, and His172 were present in the binding pocket. SARS-CoV-2 coronavirus main protease quest grid has been recognized as center $x:-10.656$, center $y: 17.223$, and center $z: 67.024$ in dimensions $x: 20, y: 20$, and $z: 20$ in $1.0 \AA$ spacing. The importance of completeness was set to eight. The additional restrictions were fixed and not specified by default for AutoDock Vina. The compound which consumes the smallest binding affinity value is the best score compound, and the implications have been visually analyzed with Discovery Studio 2019.

\section{Results and Discussion}

\subsection{Characterization of Silver Nanoparticles}

3.1.1. Transmission Electron Microscopy (TEM). TEM analysis was used to describe the morphology and particle size of AgNPs. Figure 3 depicts an outline of green synthesized AgNPs, which demonstrates the NPs' spherical morphology and the average size of $20 \mathrm{~nm}$. The nanoparticles are well distributed, and sizes are in the range of 0-25 $\mathrm{nm}$. Therefore, the TEM analysis clearly indicates high quality and smaller size AgNps.

3.1.2. Scanning Electron Microscopy (SEM). The SEM examination revealed well-defined uniformly spherical AgNPs with no agglomeration and a small scale. Dipteryx odorata has a lot of charged surfaces, which makes it suitable for binding metal ions from their aqueous precursor solution. Figure 4 shows an SEM representation of AgNOs.

3.1.3. Recovery of Catalyst. In the biosynthetic phase, catalyst recovery is critical. We have studied the recyclability of the AgNPs three times with almost the same catalytic activity, by using the reaction of isatins 1,2 , and $3 a-3 n$. After adding DMF, the AgNPs were separated by centrifugation and washed with $\mathrm{EtOH}$ to eliminate the residual substance. The above procedure was repeated for three cycles.

3.2. Chemistry. The synthetic method involves the use of thiosemicarbazide for the hydrolysis of compounds 1, 2, and $3 a-3 n$. Previously reported protocols were followed to synthesize the compounds [47, 48]. AgNPs were used as the green catalysts to synthesize the target compounds. The AgNPs are cost-effective and easily available. They do not get consumed during the chemical reactions and increase the rate of the reactions. The AgNPs were more active than other noble metal-based catalysts [49, 50]. The method followed for the synthesis of the target compounds involves a green pathway. The products are obtained within a short time. The compounds were obtained in excellent yields. The pathway followed for the synthesis of the isatin derivatives 1, 2, and 3a-n are presented in Scheme 2. The optimizations of the substituent are given in Table 1. The chemical structures of the synthesized isatin derivatives are presented in Figure 5.

Fourier transform-infrared, proton nuclear magnetic resonance $\left({ }^{1} \mathrm{H} \mathrm{NMR}\right)$ spectroscopy, ${ }^{13} \mathrm{C}$ NMR spectroscopy, and mass spectrometry techniques were used to characterize 


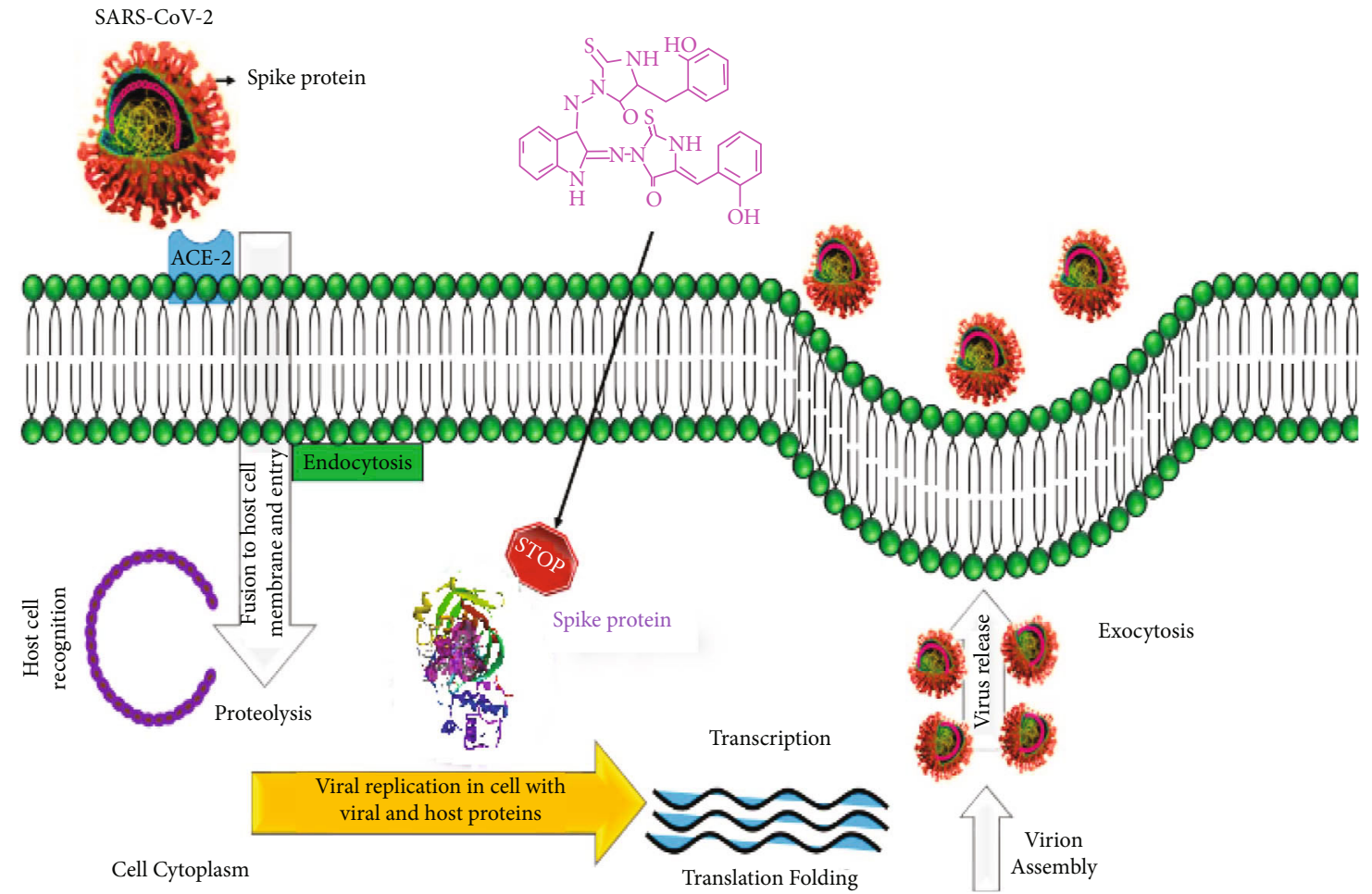

Scheme 3: Pathway mechanism of compound $3 c$ interacts with spike protein.

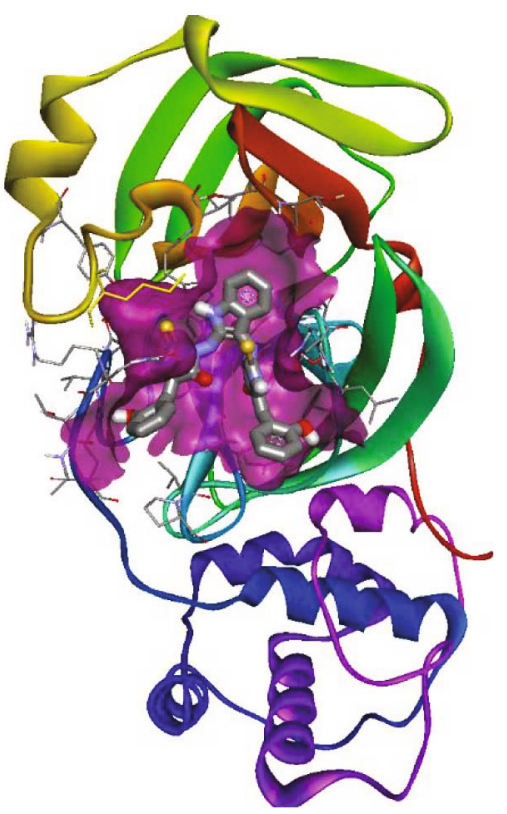

FIGURE 9: Interaction of compound $3 c$ with SARS-CoV-2 spike protein.

the compounds. A molecular ion peak was observed at $\mathrm{m} / \mathrm{z}$ $=292.06$ in the high-resolution mass spectral profile recorded for compound 1 . The FT-IR spectral profiles recorded for compounds 1,2 , and $3 \mathrm{a}-3 \mathrm{n}$ were analyzed. Absorption bands at 3475, 3421, 3170, 1699, 1682, and $1135 \mathrm{~cm}^{-1}$, corresponding to the $-\mathrm{NH}_{2},-\mathrm{NH},-\mathrm{Ar}-\mathrm{CH},-$

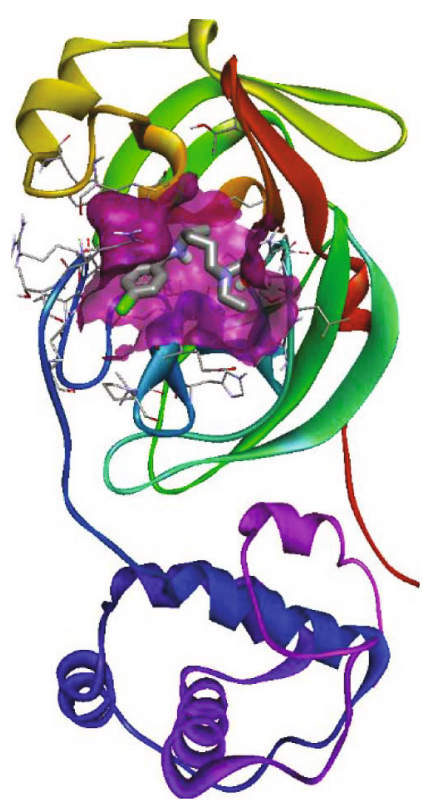

FIGURE 10: Interaction of hydroxychloroquine with SARS-CoV-2 spike protein.

$\mathrm{C}=\mathrm{O},-\mathrm{C}=\mathrm{N}$, and $-\mathrm{C}=\mathrm{S}$ groups were observed. Signals at 12.85 (corresponding to the $-\mathrm{N}-\mathrm{NH}$ unit in isatin), 11.24 (corresponding to the $-\mathrm{NH}$ unit in isatin), 6.89 (corresponding to the $\mathrm{NH}_{2}$ units in the imidazolidine ring), and 2.75 (corresponding to the $\mathrm{CH}_{2}$ units in the imidazolidine ring) ppm were observed in the ${ }^{1} \mathrm{H}-\mathrm{NMR}$ spectral profile. The 


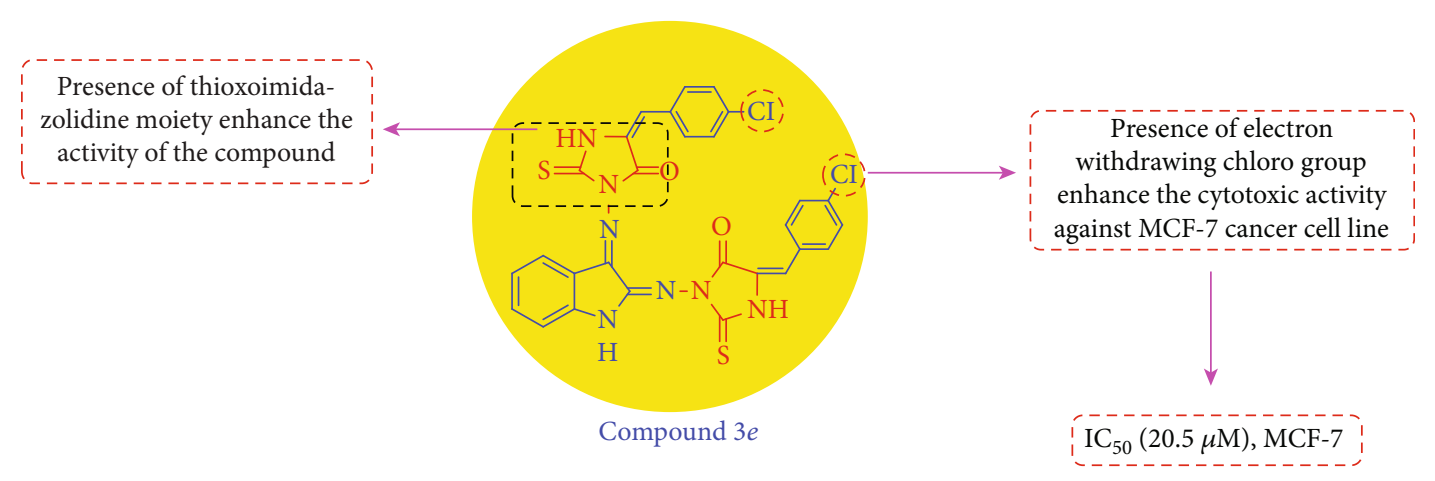

FIGURE 11: SARS-CoV-2 analysis of highly active compound 3e.

signals in the range of 7.53-6.92 were attributed to the Ar$\mathrm{CH}$ units present in the imidazolidine ring. Signals at $179.61,162.93,142.53$, and $39.13 \mathrm{ppm}$ were observed in the ${ }^{13} \mathrm{C}-\mathrm{NMR}$ spectral profile. The signals were attributed to the $-\mathrm{C}=\mathrm{S},-\mathrm{C}=\mathrm{O},-\mathrm{C}=\mathrm{N}$, and $-\mathrm{CH}_{2}$ carbon atoms present in the imidazole ring.

3.3. Cytotoxic Activity. The newly synthesized isatin conjugates $(1,2$, and $3 \mathrm{a}-3 \mathrm{n})$ were screened for their cytotoxic activity using Sulforhodamine B stain (SRB) anticancer assay (different concentrations of $10,25,50$, and $100 \mu \mathrm{M}$ at $48 \mathrm{~h}$ ) against the MCF-7 cancer cell line. Table 2 shows the different concentrations of the compounds under test $(10,25,50$, and $100 \mathrm{mM}$ ) that were added to the cell monolayer. The molar concentration required for $50 \%$ inhibition of cell viability $\left(\mathrm{IC}_{50}\right)$ was calculated and compared to the reference drug doxorubicin. The cytotoxicity of the compounds in normal breast cells (MCF-10A) and lung cells (MRC-5) was evaluated by using the MTT assay. The assay found that these compounds did not affect normal cell growth $\left(\mathrm{IC}_{50}\right.$ value $\left.>100\right)$. The in vitro cytotoxicity of indoline-2,3dione derivatives of normal cell lines is given in Table 3. The compound $3 \mathrm{e}$ is highly active (MCF-7, $\mathrm{IC}_{50}=20.5 \mu \mathrm{M}$ ), compared with standard doxorubicin and other compounds; compound $3 \mathrm{c}$ has the equipotent active (MCF-7, I $\left.\mathrm{C}_{50}=30.1 \mu \mathrm{M}\right)$; and compound $3 \mathrm{~d}$ has moderately active (MCF-7, $\left.\quad \mathrm{IC}_{50}=25.3 \mu \mathrm{M}\right)$. Hence, compound $3 \mathrm{e}$ $\left(\mathrm{IC}_{50}=20.5 \mu \mathrm{M}\right)$ was relatively more effective than the other compounds. Finally, these compounds have exposed their safety to normal cells, and compound $3 \mathrm{e}$ can be used as a lead compound to further develop more effective drugs for the MCF-7 (breast) cancer cell line. The cytotoxic sequence of the synthetic compounds is given in Figure 6.

3.4. Docking Studies. Docking expressions were carried out in order to gain a better understanding of the biological activity mechanism. The compounds 1,2 , and $3 \mathrm{a}-3 \mathrm{n}$ were analyzed by the AutoDock Vina for their docking behaviour against the SARS-CoV-2 coronavirus main protease (PDB ID: 6LU7). The overall studied inhibitors indicate negative binding energy. The compound $3 \mathrm{c}$ demonstrated remarkable inhibition ability with a binding energy value of $-9.4 \mathrm{kcal} /$ mol over other compounds $1(-6.1 \mathrm{kcal} / \mathrm{mol}), 2(-7.3 \mathrm{kcal} /-$ $\mathrm{mol}), \quad 3 \mathrm{a} \quad(-7.8 \mathrm{kcal} / \mathrm{mol}), \quad 3 \mathrm{~b} \quad(-8.9 \mathrm{kcal} / \mathrm{mol}), \quad 3 \mathrm{~d}$
$(-8.7 \mathrm{kcal} / \mathrm{mol}), \quad 3 \mathrm{e}(-8.2 \mathrm{kcal} / \mathrm{mol}), \quad 3 \mathrm{f}(-8.1 \mathrm{kcal} / \mathrm{mol}), \quad 3 \mathrm{~g}$ $(-7.8 \mathrm{kcal} / \mathrm{mol}), 3 \mathrm{~h}(-6.2 \mathrm{kcal} / \mathrm{mol}), 3 \mathrm{i}(-7.2 \mathrm{kcal} / \mathrm{mol}), 3 \mathrm{j}$ $(-7.8 \mathrm{kcal} / \mathrm{mol}), 3 \mathrm{k}(-8.3 \mathrm{kcal} / \mathrm{mol}), 3 \mathrm{l}(-8.1 \mathrm{kcal} / \mathrm{mol}), 3 \mathrm{~m}$ $(-7.2 \mathrm{kcal} / \mathrm{mol})$, and $3 \mathrm{n}(-7.9 \mathrm{kcal} / \mathrm{mol})$ and hydroxychloroquine $(-6.2 \mathrm{kcal} / \mathrm{mol})$ in the target protein. The approvable bond distance between $\mathrm{H}$-acceptor and $\mathrm{H}$-donor atoms is less than $3.5 \AA$, which plays an important role in ligand binding stability [51]. The distance between hydrogenbound compounds in the target protein 6LU7 was less than $3.5 \AA$, indicating that the protein and ligands were forming a stable hydrogen bond. The 6LU7 receptor has five hydrogen bonding interactions with the compound $3 \mathrm{c}$. The amino acid residues Phe140, Asn142, His164, Arg188, and Thr190 were entangled by the hydrogen linkage, which had bond lengths of 2.65, 2.62, 3.19, 2.66, and 2.49 A. His41, Leu141, Cys145, Met165, and Glu166 amino acid residues were in hydrophobic contact. Figure 7 shows the hydrogen bonding and hydrophobic contacts of amino acid residues in the 6LU7 protein with compound $3 \mathrm{c}$. The compound hydroxychloroquine displays three hydrogen bonding associations with the 6LU7 receptor. The amino acid residues of Ser144, Cys145, and His164 were entangled through the relationship of the hydrogen linkage with bond lengths between 2.43, 2.62, and 2.66 ̊. His41, Leu141, and Met165 amino acid residues were in hydrophobic contact, with the compound hydroxychloroquine; Figure 8 shows hydrogen bonding and hydrophobic contacts in 6LU7 protein amino acid residues. Compound $3 \mathrm{c}$ had a higher ability to inhibit SARSCoV-2 coronavirus key protease (PDB ID: 6LU7) than other compounds, according to the results. The findings are summarized in Table 4. The pathway mechanism of compound $3 \mathrm{c}$ and its interactions with the spike protein are illustrated in Scheme 3. The interaction of compound $3 c$ and hydroxychloroquine with the SARS-CoV-2 spike protein is given in Figures 9 and 10.

3.5. Structure Activity Relationship. The highly active isatin derivatives of compound $3 e$ and its SARS-CoV-2 are shown in Figure 11. The biological data of the selected isatin derivatives 1,2 , and $3 \mathrm{a}-3 \mathrm{n}$ evidenced that compound $3 \mathrm{e}$ is the most efficacious (MCF-7, IC $\left._{50}(20.5 \mu \mathrm{M})\right)$ than control doxorubicin. Due to the presence of the thioxoimidazolidine ring fused with chlorobenzaldehyde, it was established to have high cytotoxic activity against the MCF-7 cancer cell 
line. The reason is that chlorine is an electron withdrawing group and is present in the paraposition on the benzene ring connected to the thioxoimidazolidine backbone. The effect of 4-chloro substitution was favorable to the activities of the compounds. The substitution of both phenyl rings enhances the activity of the compound $3 \mathrm{e}$. The remaining compounds exhibited weak cytotoxicity to the MCF-7 cancer cell line.

\section{Conclusions}

We have developed an environmentally friendly green synthetic method for the synthesis of AgNPs using Dipteryx odorata. A simple method was followed for the reduction of the silver ions to produce AgNPs. The NPs exhibited excellent catalytic activities during the preparation of compounds 1,2 , and $3 a-3 n$ in ethanol. The synthetic method is simple, green, cost-effective, and easy to implement. The compounds could be synthesized in high yields within a short time. The cytotoxic activities of the newly synthesized isatin conjugates $(1,2$, and $3 a-3 n)$ were determined. The in silico molecular docking technique was also used to study the compounds. Compound $3 \mathrm{e}$ was found to be the most effective against the MCF-7 cancer cell line $\left(\mathrm{IC}_{50}=20.5 \mu \mathrm{M}\right.$ ). The activity was comparable to the activities of the standard doxorubicin and other compounds. Satisfactory docking scores were obtained when compounds 1,2 , and $3 a-3 n$ were studied. The interaction residues present in the spike protein of SARS-CoV-2 (PDB ID: 6LU7) were identified. A significantly high binding affinity $(-9.4 \mathrm{kcal} / \mathrm{mol})$ was recorded for compound $3 \mathrm{c}$ when hydroxychloroquine was used as a standard. The binding affinity recorded for compound $3 c$ was higher than the binding affinities recorded for the other compounds. The synthesized compound $3 \mathrm{e}$ is a potential lead molecule that can be used to treat cancer. Compound $3 \mathrm{c}$ can be potentially used to develop antiviral drugs for the treatment of COVID-19.

\section{Data Availability}

The supplementary file data used to support the findings of this study are included within the supplementary information file (available here).

\section{Conflicts of Interest}

The authors declare no conflict of interest.

\section{Acknowledgments}

This research work was funded by Researchers supporting Project number (RSP-2021/27), King Saud University, Saudi Arabia.

\section{Supplementary Materials}

The supplementary material used to support the findings of this study is included within the supplementary information files. (The supplementary file contains ${ }^{1} \mathrm{H}-\mathrm{NMR}$ and ${ }^{13} \mathrm{C}$ NMR spectra.) (Supplementary Materials)

\section{References}

[1] World Health Organization, GCM teleconference-note for the records, 2020, Subject: pneumonia in Wuhan, China.

[2] D. S. C. Hui and A. Zumla, "Severe Acute Respiratory Syndrome: Historical, Epidemiologic, and Clinical Features," Infectious Disease Clinics of North America, vol. 33, no. 4, pp. 869-889, 2019.

[3] T. K. Burki, “The Russian vaccine for COVID-19," Respiratory Medicine, vol. 8, no. 11, pp. e85-e86, 2020.

[4] WHO, "Coronavirus disease (COVID-19) dashboard," https:// covid19.who.int/accessed on 2nd Feb, 2021.

[5] S. A. Amin and T. Jha, "Fight against novel coronavirus: a perspective of medicinal chemists," European Journal of Medicinal Chemistry, vol. 201, p. 112559, 2020.

[6] S. A. Amin, K. Ghosh, S. Gayen, and T. Jha, "Chemical-informatics approach to COVID-19 drug discovery: Monte Carlo based QSAR, virtual screening and molecular docking study of somein-housemolecules as papain-like protease (PLpro) inhibitors," Journal of Biomolecular Structure \& Dynamics, vol. 39, no. 13, pp. 4764-4773, 2021.

[7] T. Pillaiyar, S. Meenakshisundaram, and M. Manickam, "Recent discovery and development of inhibitors targeting coronaviruses," Drug discovery today, vol. 25, no. 4, pp. 668688, 2020.

[8] K. Ghosh, S. A. Amin, S. Gayen, and T. Jha, "Chemical-informatics approach to COVID-19 drug discovery: exploration of important fragments and data mining based prediction of some hits from natural origins as main protease (Mpro) inhibitors," Journal of Molecular Structure, vol. 1224, p. 129026, 2020.

[9] R. A. Khailany, M. Safdar, and M. Ozaslan, "Genomic characterization of a novel SARS-CoV-2," Gene Reports, vol. 16, p. 100682, 2020.

[10] R. Yu, L. Chen, R. Lan, R. Shen, and P. Li, "Computational screening of antagonists against the SARS-CoV-2 (COVID19) coronavirus by molecular docking," International Journal of Antimicrobial Agents, vol. 56, no. 2, p. 106012, 2020.

[11] Y. Zhou, Y. Hou, J. Shen, Y. Huang, W. Martin, and F. Cheng, "Network-based drug repurposing for novel coronavirus 2019nCoV/SARS-CoV-2," Cell Discovery, vol. 6, no. 1, pp. 1-8, 2020.

[12] I. Willner, R. Baron, and B. Willner, "Growing metal nanoparticles by enzymes," Adv. Maternité, vol. 18, no. 9, pp. 11091120, 2006.

[13] V. C. Verma, R. N. Kharwar, and A. C. Gange, "Biosynthesis of antimicrobial silver nanoparticles by the endophytic fungus Aspergillus clavatus," Nanomedicine, vol. 5, no. 1, pp. 33-40, 2010.

[14] S. S. Shankar, A. Rai, A. Ahmad, and M. Sastry, "Rapid synthesis of $\mathrm{Au}, \mathrm{Ag}$, and bimetallic Au core-Ag shell nanoparticles using Neem (_Azadirachta indica_) leaf broth," Journal of Colloid and Interface Science, vol. 275, no. 2, pp. 496-502, 2004.

[15] C. Krishnaraj, E. G. Jagan, S. Rajasekar, P. Selvakumar, P. T. Kalaichelvan, and N. Mohan, "Synthesis of silver nanoparticles using _Acalypha indica_ leaf extracts and its antibacterial activity against water borne pathogens," Colloids and Surfaces B: Biointerfaces, vol. 76, no. 1, pp. 50-56, 2010.

[16] A. Mohammed Fayaz, M. Girilal, R. Venkatesan, and P. T. Kalaichelvan, "Biosynthesis of anisotropic gold nanoparticles using _Maduca longifolia_ extract and their potential in 
infrared absorption," Colloids and Surfaces. B, Biointerfaces, vol. 88, no. 1, pp. 287-291, 2011.

[17] H. Hirata, Y. Hinoda, V. Shahryari et al., "Long noncoding RNA MALAT1 promotes aggressive renal cell carcinoma through Ezh2 and interacts with miR-205," Cancer Research, vol. 75, no. 7, pp. 1322-1331, 2015.

[18] X. Fu, Y. Liu, C. Zhuang, L. Liu, Z. Cai, and W. Huang, "Synthetic artificial microRNAs targeting UCA1-MALAT1 or cMyc inhibit malignant phenotypes of bladder cancer cells T24 and 5637," Molecular BioSystems, vol. 11, no. 5, pp. 1285-1289, 2015.

[19] T. Han, F. Jiao, H. Hu et al., "EZH2 promotes cell migration and invasion but not alters cell proliferation by suppressing E-cadherin, partly through association with MALAT-1 in pancreatic cancer," Oncotarget, vol. 7, no. 10, pp. 11194-11207, 2016.

[20] M. T. Qiu, J. W. Hu, R. Yin, and L. Xu, "Long noncoding RNA: an emerging paradigm of cancer research," Tumor Biology, vol. 34, no. 2, pp. 613-620, 2013.

[21] X. Lu, C. Cheng, G. Wang, X. Shu, J. Ma, and Q. Tong, "Synergistic enhancement of cancer therapy using a combination of fusion protein MG7-scFv/SEB and tumor necrosis factor alpha," Protein and Peptide Letters, vol. 20, no. 4, pp. 467472, 2013.

[22] A. A. Imani-Fooladi, F. Yousefi, S. F. Mousavi, and J. Amani, "In silico design and analysis of TGF $\alpha$ L3-SEB fusion protein as "a new antitumor agent" candidate by ligand-targeted superantigens technique," Iranian Journal of Cancer Prevention, vol. 7, no. 3, pp. 152-164, 2014.

[23] I. Adkins, L. Sadilkova, and L. Palova-Jelinkova, Bacterial toxins in cancer immunotherapy. Forum on immunopathological diseases and therapeutics, Begel House Inc, 2013.

[24] Y. Han, S. Li, X. Cao et al., "Different Inhibitory Effect and Mechanism of Hydroxyapatite Nanoparticles on Normal Cells and Cancer Cells_In Vitro_and_In Vivo_,'Scientific Reports, vol. 4, no. 1, pp. 7134-7141, 2014.

[25] J. Ferlay, I. Soerjomataram, R. Dikshit et al., "Cancer incidence and mortality worldwide: sources, methods and major patterns in GLOBOCAN 2012," International Journal of Cancer, vol. 136, no. 5, pp. E359-E386, 2015.

[26] C. Melis, S. Distinto, G. Bianco et al., "Targeting tumor associated carbonic anhydrases IX and XII: highly isozyme selective coumarin and psoralen inhibitors," ACS Medicinal Chemistry Letters, vol. 9, no. 7, pp. 725-729, 2018.

[27] C. Melis, R. Meleddu, A. Angeli et al., "Isatin: a privileged scaffold for the design of carbonic anhydrase inhibitors," Journal of Enzyme Inhibition and Medicinal Chemistry, vol. 32, no. 1, pp. 68-73, 2017.

[28] G. Bianco, R. Meleddu, S. Distinto et al., "N-Acylbenzenesulfonamide dihydro-1,3,4-oxadiazole hybrids: seeking selectivity toward carbonic anhydrase isoforms," ACS Medicinal Chemistry Letters, vol. 8, no. 8, pp. 792-796, 2017.

[29] R. Meleddu, E. Maccioni, S. Distinto et al., "New 4-[(3-cyclohexyl-4-aryl-2,3-dihydro-1,3-thiazol-2-ylidene)amino]benzene-1-sulfonamides, synthesis and inhibitory activity toward carbonic anhydrase I, II, IX, XII," Bioorganic \& Medicinal Chemistry Letters, vol. 25, no. 16, pp. 3281-3284, 2015.

[30] R. Meleddu, S. Distinto, F. Cottiglia et al., "Tuning the dual inhibition of carbonic anhydrase and cyclooxygenase by dihydrothiazole benzensulfonamides," ACS Medicinal Chemistry Letters, vol. 9, no. 10, pp. 1045-1050, 2018.
[31] R. J. Motzer, M. D. Michaelson, B. G. Redman et al., “Activity of SU11248, a multitargeted inhibitor of vascular endothelial growth factor receptor and platelet-derived growth factor receptor, in patients with metastatic renal cell carcinoma," Journal of Clinical Oncology, vol. 24, no. 1, pp. 16-24, 2006.

[32] H. Prenen, J. Cools, N. Mentens et al., "Efficacy of the kinase inhibitor SU11248 against gastrointestinal stromal tumor mutants refractory to imatinib mesylate," Clinical Cancer Research, vol. 12, no. 8, pp. 2622-2627, 2006.

[33] R. Griffith, M. N. Brown, A. McCluskey, and L. K. Ashman, "Small molecule inhibitors of protein kinases in cancer- how to overcome resistance," Mini Reviews in Medicinal Chemistry, vol. 6, no. 10, pp. 1101-1110, 2006.

[34] F. M. Klenke, A. Abdollahi, E. Bertl et al., "Tyrosine kinase inhibitor SU6668 represses chondrosarcoma growth via antiangiogenesis in vivo," BMC Cancer, vol. 7, no. 1, p. 49, 2007.

[35] P. L. Mccormack, "Nintedanib: first global approval," Drugs, vol. 75, no. 1, pp. 129-139, 2015.

[36] G. J. Roth, A. Heckel, F. Colbatzky et al., "Design, synthesis, and evaluation of indolinones as triple angiokinase inhibitors and the discovery of a highly specific 6-methoxycarbonylsubstituted indolinone (BIBF 1120)," Journal of Medicinal Chemistry, vol. 52, no. 14, pp. 4466-4480, 2009.

[37] M. Rita, P. Vilma, D. Simona, A. Antonella, and A. Rossella, "Investigating the anticancer activity of isatin/dihydropyrazole hybrids," ACS Medicinal Chemistry Letters, vol. 10, pp. 571$576,2019$.

[38] D. J. Bauer, "Clinical experience with the antiviral drug MARBORAN ${ }^{\circledR}$ (1-METHYLISATIN 3-THIOSEMICARBAZONE) *," Annals of the New York Academy of Sciences, vol. 130, no. 1, pp. 110-117, 1965.

[39] T. B. Johnson and W. M. Scott, "Hydantoins: the synthesis of 2-thiohydantoins from acyl derivatives of $\alpha$-amino acids," The Journal of the American Chemical Society, vol. 35, no. 9, pp. 1208-1213, 1913.

[40] N. Karalí, A. Gürsoy, N. Terzioglu, S. Özkírímlí, H. Özer, and A. C. Ekinci, "Synthesis and preliminary CNS depressant activity evaluation of new 3-[(3-substituted-5-methyl-4-thiazolidinon-2-ylidene)hydrazono]-1H-2-indolinones and 3[(2-thioxo-3-substituted-4,5-imidazolidine-dion-1-yl)imino]1H-2-indolinones," Archiv der Pharmazie, vol. 331, no. 7-8, pp. 254-258, 1998.

[41] M. E. Voss, P. H. Carter, A. J. Tebben et al., "Both 5-arylidene2-thioxodihydropyrimidine-4,6(1_H_, $\left.{ }_{-} \mathrm{H}_{-}\right)$-diones and 3thioxo-2,3-dihydro-1 __H_ -imidazo[1,5- _a_ ]indol-1-ones are light-Dependent tumor necrosis factor- $\alpha$ antagonists," Bioorganic \& Medicinal Chemistry, vol. 13, no. 3, pp. 533538, 2003.

[42] K. Tachibana, I. Imaoka, T. Shiraishi et al., "Discovery of an orally-active nonsteroidal androgen receptor pure antagonist and the structure-activity relationships of its derivatives," Chemical \& Pharmaceutical Bulletin, vol. 56, no. 11, pp. 1555-1561, 2008.

[43] G. G. Muccioli, J. Wouters, C. Charlier et al., "Synthesis and activity of 1,3,5-Triphenylimidazolidine-2,4-diones and 1,3,5triphenyl-2-thioxoimidazolidin-4-ones: characterization of new CB1Cannabinoid receptor inverse agonists/antagonists," Journal of Medicinal Chemistry, vol. 49, no. 3, pp. 872-882, 2006.

[44] A. A. el-Barbary, A. I. Khodair, E. B. Pedersen, and C. S. Nielsen, "S-Glucosylated hydantoins as new antiviral agents," 
Journal of Medicinal and Pharmaceutical Chemistry, vol. 37, no. 1, pp. 73-77, 1994.

[45] P. Skehan, R. Storeng, D. Scudiero et al., "New colorimetric cytotoxicity assay for anticancer-drug screening," Journal of the National Cancer Institute, vol. 82, no. 13, pp. 1107-1112, 1990.

[46] O. Trott and A. J. Olson, "AutoDock Vina: improving the speed and accuracy of docking with a new scoring function, efficient optimization, and multithreading," Journal of Computers \& Chemistry, vol. 31, pp. 455-461, 2009.

[47] A. A. Samar, "Synthesis, antimicrobial and anticancer activities of some 2-thiohydantion derivatives," Indian Journal of Chemistry, vol. 56B, pp. 641-648, 2017.

[48] J. A. Hasanen, H. K. Ibrahim, M. A. Zein, and I. M. El-Deen, "Synthesis, mass spectra and antimicrobial activity of some nitrogen heterocycles," Mens Agitat, vol. 3, pp. 59-72, 2008.

[49] S. Perni, V. Hakala, and P. Prokopovich, "Biogenic synthesis of antimicrobial silver nanoparticles capped with l-cysteine," Colloids and Surfaces A: Physicochemical and Engineering Aspects, vol. 460, pp. 219-224, 2014

[50] P. R. Rathi Sre, M. Reka, R. Poovazhagi, M. Arul Kumar, and K. Murugesan, "Antibacterial and cytotoxic effect of biologically synthesized silver nanoparticles using aqueous root extract of _Erythrina indica lam_, Spectrochimica Acta Part A: Molecular and Biomolecular Spectroscopy, vol. 135, pp. 1137-1144, 2015.

[51] M. Taha, N. H. Ismail, A. Khan et al., "Synthesis of novel derivatives of oxindole, their urease inhibition and molecular docking studies," Bioorganic \& Medicinal Chemistry Letters, vol. 25, no. 16, pp. 3285-3289, 2015. 